1 Title: Encoding of odors by mammalian olfactory receptors

2 Authors: Aashutosh Vihani ${ }^{1}$, Maira H. Nagai ${ }^{2}$, Conan Juan², Claire A. de March², Xiaoyang S. Hu ${ }^{2}$, John

3 Pearson $^{1,3}$, Hiroaki Matsunami ${ }^{1,2,3^{*}}$

4 1. Department of Neurobiology, Neurobiology graduate program, Duke University Medical Center, Durham, NC 27710, USA

2. Department of Molecular Genetics and Microbiology, Duke University Medical Center, Durham, NC 27710, USA

3. Duke Institute for Brain Sciences, Duke University, Durham, NC 27710, USA

*Correspondence to HM (hiroaki.matsunami@duke.edu)

\title{
11 Contributions:
}

$12 \mathrm{AV}, \mathrm{MHN}, \mathrm{XSH}$, and $\mathrm{HM}$ did in vivo experiments. CJ and CAdM did in vitro experiments. AV analyzed

13 data. CAdM did OR homology modeling. JP advised data modeling analysis. AV drafted the paper. All

14 authors reviewed and edited the paper. HM supervised the work.

16 Acknowledgements: We thank Michael Schmuker for critical reading and comments, Mengjue Jessica

17 Ni for expert technical assistance, Alex Koulakov for helpful discussions on data analysis, Priya Meesa

18 for manuscript edits, and members of the Matsunami lab for helpful discussions. AV thanks Jack J. Zhan

19 for advising how to analyze data in python during initial stages.

21 Funding Sources: This work was funded by NIH (DC014423 and DC016224) and NSF (1556207 and

22 1555919) to HM, and NIH (DC018333) to CAdM. 
bioRxiv preprint doi: https://doi.org/10.1101/2021.12.27.474279; this version posted December 28, 2021. The copyright holder for this preprint (which was not certified by peer review) is the author/funder. All rights reserved. No reuse allowed without permission.

23

24 Competing Interests: HM has received royalties from ChemCom, has received research grants from

25 Givaudan, and has received consultant fees from Kao. 


\section{Abstract:}

27 1. Identified ligands for $>500$ mouse ORs

28 2. ORs are specifically tuned towards individual odorants and their molecular properties

3. Odor molecular properties are informative of odor responses

4. Predictive modeling and convergent evolution analyses suggest specific residues within a canonical location for odorant binding

32 Olfactory receptors (ORs) constitute the largest multi-gene family in the mammalian genome, with

33 hundreds to thousands of loci in humans and mice respectively ${ }^{1}$. The rapid expansion of this massive

34 family of genes has been generated by numerous duplication and diversification events throughout

35 evolutionary history. This size, similarity, and diversity has made it challenging to define the principles

36 by which ORs encode olfactory stimuli. Here, we performed a broad surveying of OR responses, using

37 an in vivo strategy, against a diverse panel of odorants. We then used the resulting interaction profiles

38 to uncover relationships between OR responses, odorants, odor molecular properties, and OR

39 sequences. Our data and analyses revealed that ORs generally exhibited sparse tuning towards

40 odorants and their molecular properties. Odor molecular property similarity between pairs of odorants

41 was informative of odor response similarity. Finally, ORs sharing response to an odorant possessed

42 amino acids at poorly conserved sites that exhibited both, predictive power towards odorant selectivity

43 and convergent evolution. The localization of these residues occurred primarily at the interface of the

44 upper halves of the transmembrane domains, implying that canonical positions govern odor selectivity

45 across ORs. Altogether, our results provide a basis for translating odorants into receptor neuron

46 responses for the unraveling of mammalian odor coding. 


\section{Introduction:}

48 Stimulus encoding and feature extraction are fundamental tasks performed by all sensory systems.

49 Therefore, a central problem in neurobiology is defining how aspects of a stimulus are represented by

50 the activity of sensory receptors ${ }^{2-5}$. This problem is particularly intriguing in the case of olfactory

51 stimuli, which do not vary along a single, continuous dimension, such as wavelength or amplitude.

52 Odorants, rather, have discrete molecular structures that determine their physical-chemical properties.

53 An inability to relate how these discrete molecular structures and their associated physical-chemical

54 properties influence receptor responses represents a major gap in knowledge. Consequently, one

55 cannot robustly predict the neural activity patterns nor the perceptual attributes ${ }^{6}$ of an odorant

56 starting from its physical-chemical properties.

A major hindrance in deciphering the coding of olfactory information by olfactory receptors (ORs) has

been the historic inability to comprehensively identify ORs that respond to an odorant. Various in vivo,

60 ex vivo, and in vitro methods have generally suffered from either a lack of insight into receptor identity

61 or have been too low throughput for a comprehensive surveying of OR selectivity ${ }^{5,7-9}$. With the mouse

62 genome encoding over 1000 intact ORs, and odor reception following a combinatorial coding scheme,

63 where one OR can be activated by a set of odorants and one odorant can activate a combination of

64 ORs, defining a logic for peripheral odor coding is dependent on a comprehensive surveying while

65 tracking receptor identity over a large odor panel ${ }^{1,10-12}$.

67 Here, we performed a broad surveying of odorants in vivo to identify odorant-OR interactions in Mus

68 musculus. By leveraging phosphorylated S6 ribosomal subunit capture (pS6-IP) coupled to RNA-Seq

69 (pS6-IP-Seq), we were able to identify ORs expressed by recently active olfactory sensory neurons

70 (OSNs; receptor deorphanization) $)^{11,13-15}$. Then, using a library of molecular property descriptors, we 
71 parameterized the physical-chemical properties of the tested odorants to uncover relationships to the

72 responses they elicited from cognate receptors. Finally, using our data, we asked 1) how well does

73 odor molecular property similarity predict receptor response similarity and 2) if there are specific

74 amino acid positions that influence odorant selectivity amongst receptors. Our results and analyses

75 provide a foundational framework for understanding the molecular logic by which the quality of an

76 odor molecule is encoded across a mammalian receptor repertoire. 


\section{Results:}

\section{Estimation of chemical and receptor space sampling}

79 First, we set out to identify ORs activated by a set of 61 odorants at various concentrations by

80 leveraging pS6-IP-Seq. Immunoprecipitation of phosphorylated ribosomes from activated neurons

81 followed by associated mRNA profiling by RNA-Seq, and differential expression analysis, enabled us to

82 identify ORs expressed by OSNs activated by specific odorants (Supplementary figure 1A) ${ }^{11,14,15}$. ORs

83 were considered odor-responsive if enrichment values $\left(\log _{2} \mathrm{FC}\right)$ were positive with a false discovery

84 rate $(F D R)<0.05$. Considering all odorants at all tested concentrations, this approach deorphanized a

85 total of 555 ORs across 72 conditions (Supplementary table 1). Considering unique odorants yielding at

86 least one activated OR at the lowest tested concentration, this approach deorphanized a total of 375

87 ORs across 52 odorants.

89 To examine the bias in our odorant set, we built an 1811-dimensional (1811D) space in which each

90 dimension represented a molecular property descriptor ${ }^{5}$, such as molecular weight, number of atoms,

91 or aromatic ratio, parameterizing the physical-chemical properties of the odor molecule. We then

92 plotted our 52 uniquely tested odorants together with 4680 other small molecules ${ }^{16}$ other small

93 molecules commonly found in foods and fragrances in this 1811D space to construct a chemical space

94 consisting of a total of 4732 small molecules. Visualization of the first two principal components (PCs)

95 did not reveal any obvious segregation of the test odorants, suggesting a broad sampling of chemical

96 space by our test odor panel (Figure 1A, Supplementary figure 1B-C). To examine bias in our resulting

97 deorphanized OR cohort, we computed pairwise OR Grantham distances ${ }^{17}$, an index of amino acid

98 similarity, and visualized the results using multidimensional scaling (MDS). Examination of the first two

99 MDS coordinates did not reveal any obvious segregation of the deorphanized 375 ORs, suggesting a

100 broad sampling of receptor space (Figure 1B). 
A

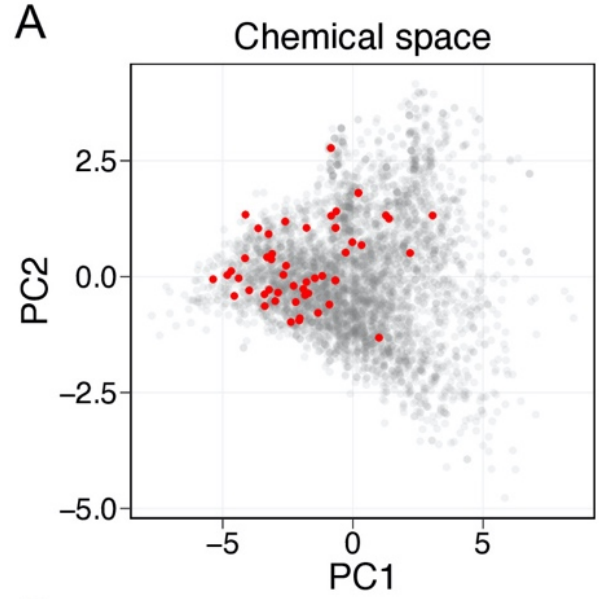

C

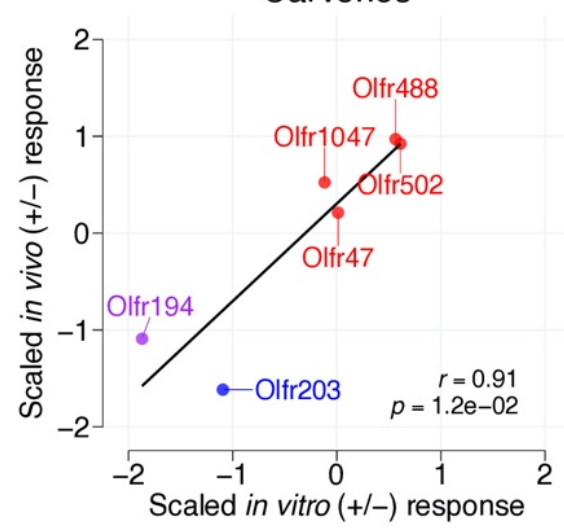

B

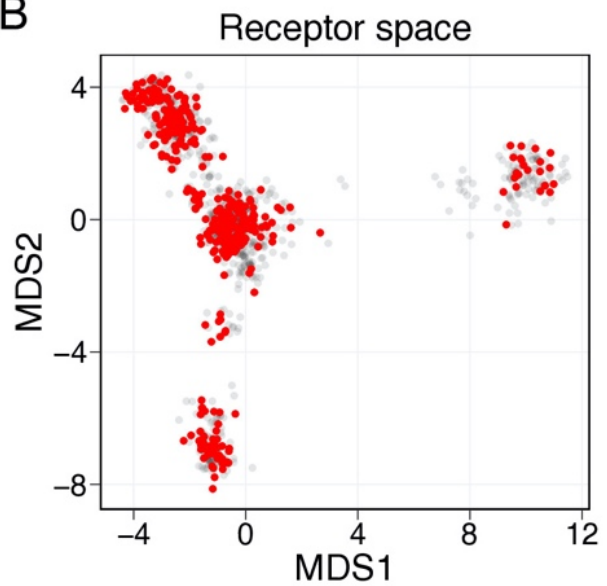

Menthols

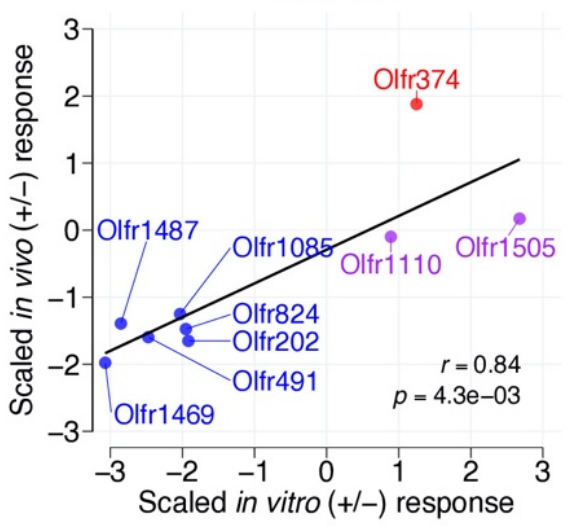

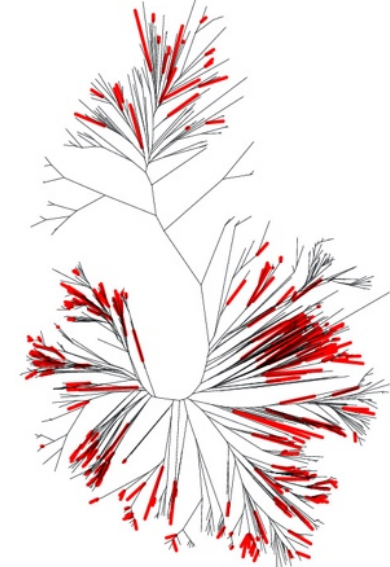

2-Octanols

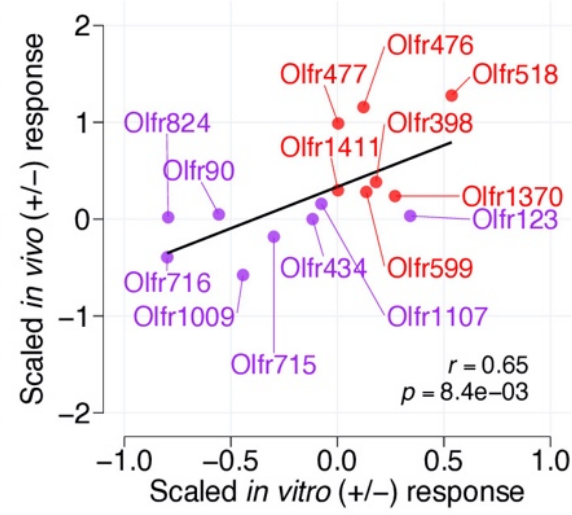

Figure 1. Data bias and pS6-IP-Seq validation. A, A total of 1811 molecular descriptors were calculated for a total of 4732 small molecules. The small molecules were projected onto a 2D chemical space made of the first and second principal components. The 52 unique odorants tested by pS6-IP-Seq at low concentrations, each yielding at least one activated OR, are colored in red. B, Left, Grantham distances were used to calculate a distance matrix for intact ORs. The matrix was visualized in two dimensions with multidimensional scaling to represent receptor space. ORs responding to at least one of the 52 tested odorants are colored red $(n=375)$. Right, a phylogenetic tree of intact ORs. Tree edges with identified and unidentified agonists at low concentrations are colored red and black respectively. C, ORs responsive to tested enantiomers were evaluated by heterologous expression. ORs enriched by pS6-IP-Seq against (+)-odorant are colored red while ORs enriched by (-)-odorant are colored blue. ORs enriched by both enantiomers are colored purple. Linear regression reveals in vivo and in vitro responses to be highly correlated (carvones $r=0.91, p=$ 0.012 ; menthols $r=0.84, p=0.0043$; 2-octanols $r=0.65, p=0.0084$ ).

102 To validate the receptor specificity of the pS6-IP-Seq dataset, we selected enantiomers (carvones, menthols, and 2-octanols) for in vitro testing. We transiently expressed ORs responsive to tested 

would provide an index of receptor selectivity even amongst structurally similar odorants (Figure 1C, Supplementary figure $2 A-F)$.

\section{Describing receptor tuning}

112 Having broadly sampled chemical and receptor spaces, we next sought to quantify the relative 113 responses of individual receptors to the test odor panel. Individual receptors displayed unique

114 response profiles across the odorants. Examining receptor tuning did not reveal a bimodal distribution

115 of narrowly and broadly tuned receptors, but rather a continuum of tuning breadths with an average of 1161.85 cognate odorants per significantly responding receptor (Figure 2A-D, Supplementary figure 3A-B).

118 To describe the tuning of ORs towards specific molecular properties, we next generated property 119 strength vectors (PSVs) for each of the molecular descriptors (Figure 2E) ${ }^{18}$. The responsiveness of each

120 OR to each molecular property was then characterized as a Pearson's correlation between the odor

121 response spectrum and the values taken by the PSV across the 52 odorants tested (Figure 2F). The 122 array of such correlations (hereby termed property response spectrum) taken across the molecular 123 property descriptor set defined the molecular receptive range and property tuning of each OR (Figure

$1242 \mathrm{G})$. For example, several ORs that displayed robust responses towards thiol odorants yielded tuning 125 towards the "number of thiol groups" molecular property (Supplementary figure 4A-D). The number of 126 properties that single receptors responded to significantly (FDR $<0.05$ ) varied from receptor to

127 receptor with a range of 0 to 136 (mean $=7.91$, median $=2)$. Indeed, the majority of deorphanized ORs 128 (223/375) displayed significant correlations to at least one of the molecular property descriptors 129 (Figure 2H). Within the subset of significant OR response-property pairs (2967/ 679125), correlations 


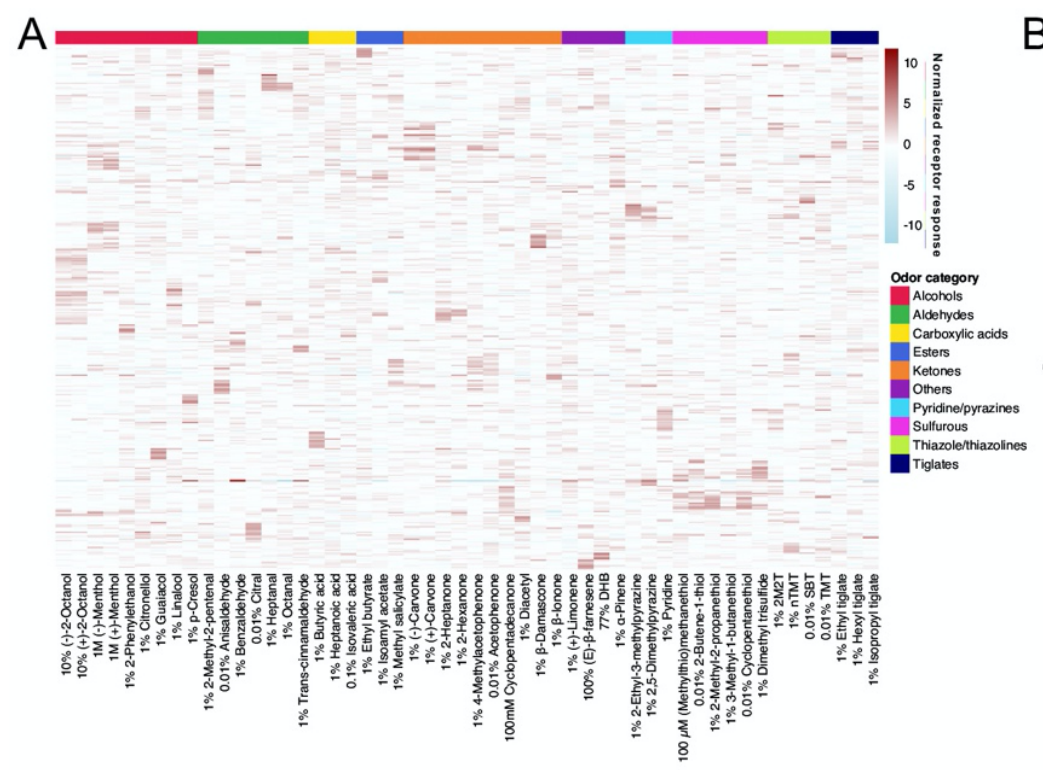

C

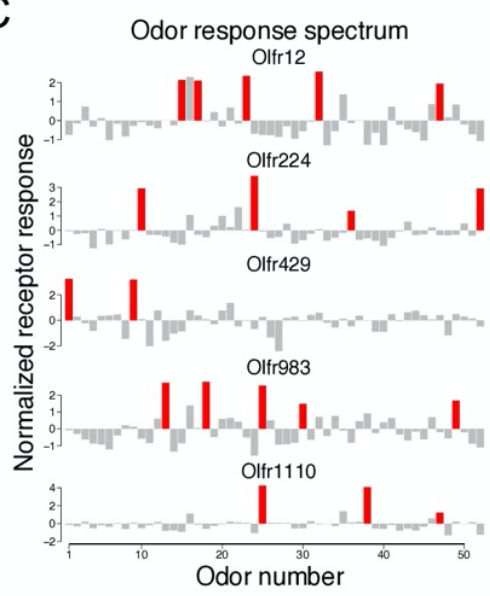

F

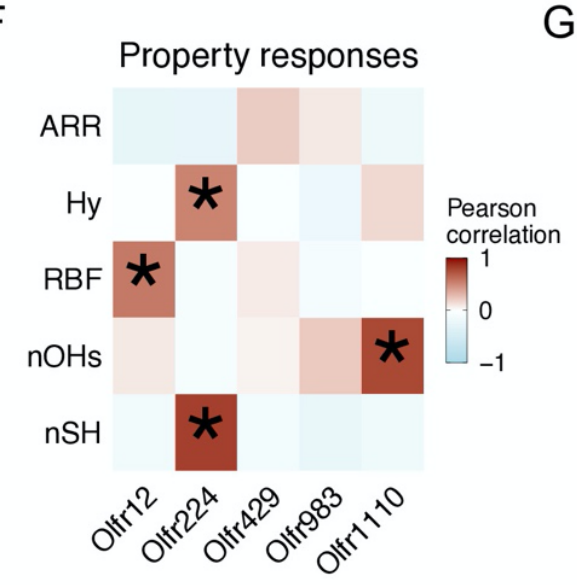

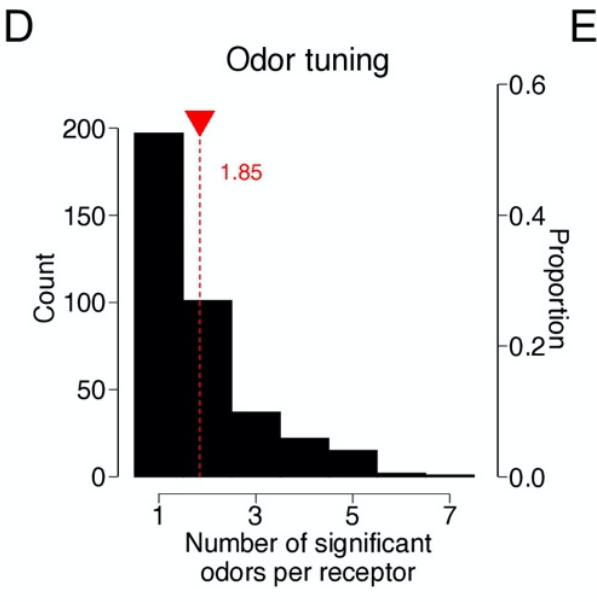

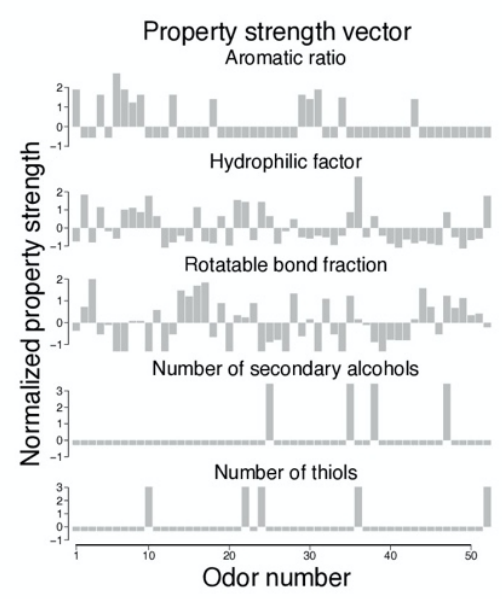

G

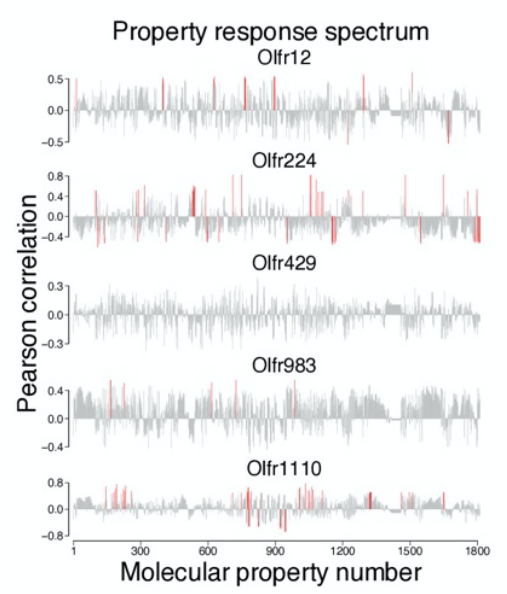

$\mathrm{H}$

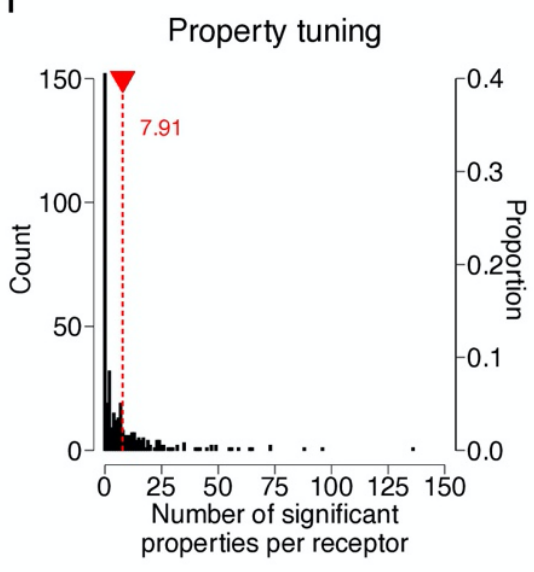

Figure 2. Combinatorial coding and OR tuning. A, Odor responses across the 52 unique odorants, tested at low concentrations, visualized by heatmap. A total of 375 ORs were determined to be responsive to at least one of the tested odorants $\left(\log _{2} F C>0\right.$ and FDR $\left.<0.05\right)$. Responses are 
normalized such that each odor has zero response mean and one standard deviation (z-scored). Responses are color coded from negative, to zero, to positive responses in light blue to white to red. The following odorants are abbreviated: 2-methyl-2-thaizoline (2M2T), 2,4,5-trimethyl-4,5dihydrothiazole (TMT), 2-sec-butyl-4,5-dihydrothiazole (SBT), 2,4,5-trimethylthiazole (nTMT), and 3,4-dehydro-exo-brevicomin (DHB). ORs were sorted by correlation distance. Odorants were sorted by odor category. B, Odor responses across the same 52 odorants visualized by chord plot. Odorants were sorted by odor category and associations were visualized. Association band thickness corresponds to the number of ORs shared, while color corresponds to overall response similarity across the 375 deorphanized ORs using correlation distance (1-r). C, Z-scored odor response spectra of five example ORs across the 52 tested odorants. Responses were normalized such that each OR is z-scored. D, Histogram of the number of significant odorants per receptor. On average, each responding OR was activated by 1.85 odorants $(n=692)$. E, Z-scored PSVs of five example molecular properties across the same set of odorants as B. F, Property responses given by the Pearson's correlation coefficients between the odor responses (B) and PSVs (D) calculated over the 52 odorants in the panel. The following molecular properties are abbreviated: aromatic ratio (ARR), hydrophilic factor $(\mathrm{Hy})$, rotatable bond fraction (RBF), number of secondary alcohols ( $\mathrm{nOHs}$ ), and number of thiols (nSH). G, Property response spectra characterized by Pearson's correlation coefficients between the five example ORs and 1811 molecular properties. $\mathbf{H}$, Histogram of the number of significant molecular properties per receptor $(n=2967)$. On average, each odorresponsive OR was significantly correlated with 7.91 molecular properties with a median of 2 (FDR < 0.05). Out of the 375 deorphanized ORs, 223 ORs displayed significant correlations to at least one molecular property descriptor.

\section{Odor molecular properties are informative of odor response patterns}

134 Having identified receptor responses to a large and diverse set of odorants, we next sought to determine the effectiveness of using odor molecular properties to predict receptor responses via similarities ${ }^{5,18,19}$. To describe the similarity between odorants in molecular property space, we calculated distances of normalized property strength values between odorant pairs. To represent odor similarity in OR response space, we similarly calculated pairwise distances between normalized receptor responses. Linear regression between odorant similarity distances and response similarity distances revealed a significant relationship $(r=0.29, p=3.4 \mathrm{E}-27$; Figure 3A, Supplementary figure 5AB), implying odor molecular properties were informative of receptor response patterns. 
145 possibility, we built a sparse regression and performed feature selection using the Least Absolute

146 Shrinkage and Selection Operator (LASSO). By varying the LASSO loss function ( $\lambda$ ) to influence the

147 number and relative contribution of the selected molecular properties, we observed improved

148 correlations with increasing numbers of weighted molecular properties. Importantly, by performing

149 odor pair cross-validation, we observed that parsimonious combinations of odor molecular properties

150 selected by LASSO yielded positive predictive abilities (optimal correlation distance odor pair cross-

151 validation $r=0.30$, Figure 3B, Supplementary figure $6 \mathrm{~A}-\mathrm{B})$. To complement these findings, we also

152 selected an "optimized" set of 65 molecular properties; which included descriptions of aromaticity,

153 functional group, and molecular geometry; that could be individually linearly decoded and regressed

154 from OR response patterns alone (Supplementary figure 7A, Supplementary table 2). Using ridge

155 regression with the "optimized" set of 65 molecular properties, we could again predict response

156 similarities from molecular property similarities (optimal correlation distance odor pair cross-validation

$157 r=0.30$, Figure 3C-D, Supplementary figure 7B-C). Altogether, we interpreted these "optimized" set of

15865 molecular property descriptors as both, being capable of explaining OR response variance, and

159 contributing to the natural statistics of odorants.

161 To further validate the predictive abilities of molecular properties and our molecular property

162 optimization, we also trained and cross-validated a feed-forward non-linear model (XGBoost). In the

163 first cross-validation scheme, we performed odor-pair cross-validation using all calculated molecular

164 properties as predictors. In the second, we limited molecular properties to the "optimized" set. In both

165 cross-validation schemes, predicting response similarities from molecular properties outperformed

166 shuffled controls (Figure 3E-F, Supplementary figure 8A-B). Altogether, these results show that odor

167 responses can be explained in part by combinations of molecular property descriptors. 

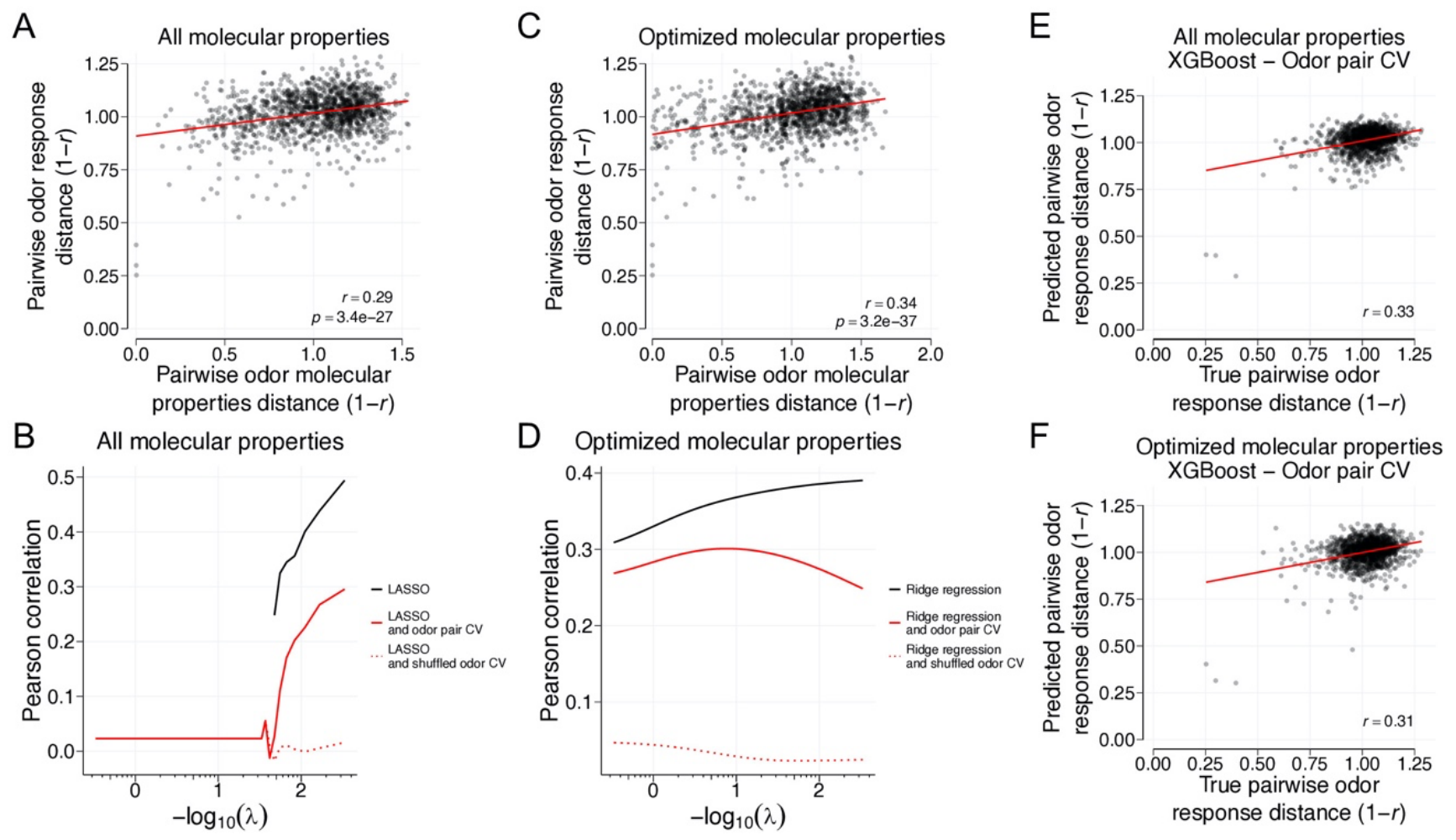

Figure 3. Pairwise odor similarity comparisons in response and property spaces. A, Pairwise correlation distance measurements between odorants in response space regressed against molecular property space $(r=0.29, p=3.4 \mathrm{E}-27, n=1326)$. B, LASSO regression as a function of varying the loss function ( $\lambda$ ). Large $\lambda$ values (leftmost edge) generally correspond to the selection of a few weighted molecular properties. Small $\lambda$ values (rightmost edge) generally correspond to the selection of many weighted molecular properties. By increasing the number of weighted molecular properties selected by the LASSO algorithm, odor responses become increasingly well fit by molecular properties (black line). To evaluate the generalizability of the sparse property selection, pairs of odorants were iteratively held-out during training and added back intact (solid red line) or shuffled (dashed red line) for cross-validation. Euclidean distances were used to quantify differences between pairs of odorants in molecular property space. Correlation distances were used to quantify differences between pairs of odorants in response space. C, Pairwise correlation distance measurements between odorants in response space against optimized molecular property space $(r=$ $0.34, p=3.2 \mathrm{E}-37, n=1326)$. $\mathrm{D}$, Ridge regression results as a function of varying the $\lambda$ loss function using the optimized set of molecular properties. Large $\lambda$ values (leftmost edge) generally correspond to dependence on a few weighted molecular properties. Small $\lambda$ values (rightmost edge) generally correspond to dependence on many weighted molecular properties. $E$, Results of odor pair crossvalidation using the XGBoost model framework with default hyperparameters with all molecular properties ( $r=0.33, n=1326)$. $\mathbf{F}$, Results of odor pair cross-validation using the XGBoost model framework with default hyperparameters with the optimized set of molecular properties $(r=0.31, n$ = 1326). 
172 logistic models using aligned ORs. For each odor fit with a regularized logistic model, receptors were

173 randomly split into $90 \%$ training and $10 \%$ testing sets for 100 repetitions. Iterating this process over the

174 set of tested odorants identified a series of weighted positions, harboring amino acids with predictive

175 power, occurring primarily at the upper halves of the fourth and fifth transmembrane domains (TMDs)

176 (Figure 4A). For example, visualizing amino acids occurring at these positions identified an enrichment

177 of cysteine and methionine residues in TMD5 amongst ORs responding to sulfurous odorants

178 (Supplementary figure 9A). Regressing the average weight assigned to each position, from odorants

179 solvable by logistic regression (area under receiver operating characteristic curve, AUROC $>0.5$ ), by

180 percent conservation revealed an anti-correlation $(r=-0.38, p=6.1 \mathrm{E}-12 \text {, Figure 4B-C })^{1}$. Using a Support

181 Vector Machine (SVM) classifier, with a linear kernel, led to similar predictions regarding the response

182 likelihoods of held-out ORs (Logistic regression AUROC $=0.70$, Linear SVM AUROC $=0.78$, Figure 4D,

183 Supplementary figure 10A).

185 The massive expansion and rapid evolution of the OR gene family posits opportunities for the

186 convergent evolution of distantly related ORs to evolve odorant selectivity independently. To search

187 for receptor sequence positions exhibiting convergent evolution, we asked if ORs sharing response to

188 an odorant possessed positions harboring amino acids with physical-chemical properties, measured by

189 Grantham's distance ${ }^{17}$, which deviated from comparable but odor-unresponsive ORs. Iterating over the

190 set of tested odorants, this analysis identified a series of poorly conserved positions $(r=-0.54, p=6.5 \mathrm{E}-$

19125 , Figure 4E, Supplementary figure 10B), especially localized to the upper half of TMD5. Regressing

192 the average weight assigned to each position, via regularized logistic regression, by the number of

193 times each position displayed convergent evolution, revealed similar findings between the two

194 approaches $(r=0.42, p=1.5 \mathrm{E}-14$, Supplementary figure 10C). Importantly, the localization of these

195 positions, and those identified by logistic models, was consistent with a region implicated in ligand 
196 binding in other class A GPCRs ${ }^{20-25}$. Altogether these results show the odorant selectivity of ORs are in

197 part explained by convergently evolving residues occurring at a common site of poorly conserved

198 residues within the TMDs.

199

200 To visualize the results of our analyses in 3D, we next built an OR homology model. Focusing on the

201 conserved "toggle switch" $Y^{6.48}$ residue previously reported to reside at the bottom of the ligand-

202 binding cavity of other class A GPCRs $20,26-28$, we consistently observed nearby residues in the upper

203 halves of TMD3, TMD5, and TMD6 as exhibiting heavy weights in our logistic models, poor

204 conservation, and convergent evolution, implying a canonical cavity for odorant binding across our

205 tested odorants. Altogether, these results are consistent with the idea that few mutations within the

206 ligand binding site of ORs can broadly reconfigure chemical tuning, a feature that is likely to have

207 facilitated the rapid evolution of receptors with distinct ligand specificities. 

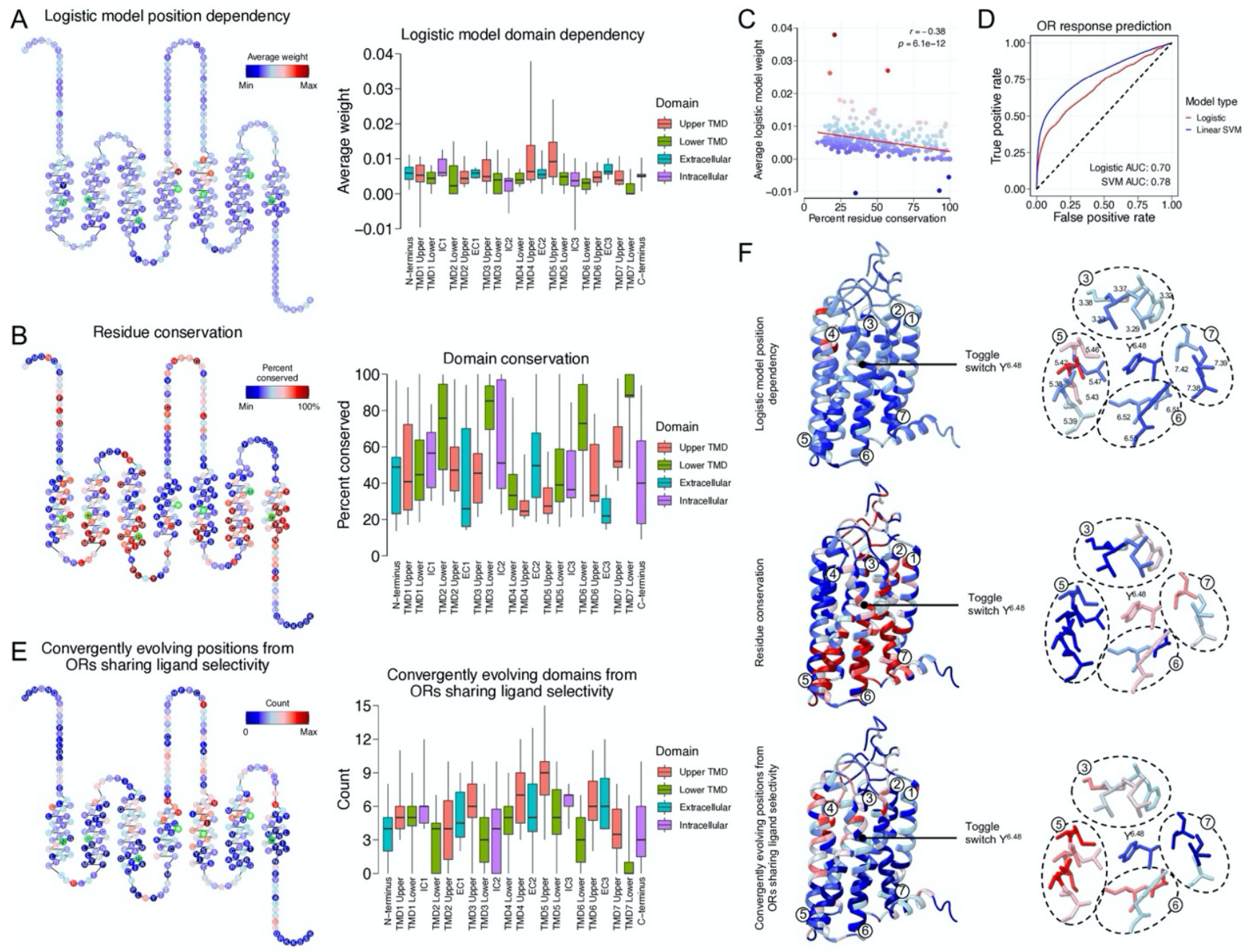

Figure 4. Sequence-function relationships of ORs. A, ORs were split into $90 \%$ training and $10 \%$ testing sets for 100 repetitions of regularized logistic regression using aligned sequences as predictors. Odorants which yielded AUROC values $>0.5$ (Supplementary table 3) were subset and non-zero weights were averaged across positions, repetitions, and odorants. Individual weights were visualized by snakeplot (left) with the most commonly occurring amino acid described at each position. Ballesteros-Weinstein x.50 numbers for each TMD are highlighted in green. Weight distributions were visualized across domains by box-and-whisker plot (right). B, Left, the most commonly occurring amino acid was quantified by its percent conservation across ORs and visualized by snake plot. Right, percent conservation distributions were visualized across receptor domains by box-and-whisker plot. C, Regressing the average weight assigned to a position, by regularized logistic regression, by its percent conservation reveals an anti-correlation $(r=-0.38, p=6.1 \mathrm{E}-12)$. $\mathrm{D}$, Using a SVM classifier with a linear kernel to predict response likelihoods of held-out ORs leads to similar results as logistic regression (logistic regression $A U R O C=0.70$, linear SVM AUROC $=0.78$ ). E, ORs sharing response to an odorant were subset and pairwise compared to a null set of ORs with comparable protein sequences. Pairwise Grantham distance distributions between the responsive and null sets were compared by Kolmogorov-Smirnov statistical test to determine if amino acid similarity distributions were different. Count measurements reflect the number of times a position harbored amino acids with differing distributions between the two groups at an FDR $<0.05$. Left, these results were visualized by snakeplot. Right, these results were visualized by box-and-whisker plot describing domain distributions. F, Left, visualization of the data in 3D using homology models. Color schemes are identical those in panels A, B, and E. TMDs are indicated. Right, zoomed in view 
bioRxiv preprint doi: https://doi.org/10.1101/2021.12.27.474279; this version posted December 28, 2021. The copyright holder for this preprint (which was not certified by peer review) is the author/funder. All rights reserved. No reuse allowed without permission.

focusing on residues found directly above the conserved $Y^{6.48}$ toggle switch residue. A consensus of poorly conserved residues found in TMD3, 5, and 6 can be seen to exhibit both higher weights by regularized logistic regression models, and convergent evolution. Ballesteros-Weinstein numbers associated with displayed residues are also described. 
Discussion:

210 Given the inordinate complexity of the chemical world, large repertoires of ORs appear to be necessary

211 for the detection and discrimination of diverse chemicals in the environment, as exemplified by the

212 significant genomic space that is systematically subjugated to ORs across numerous species. These

213 findings are compounded by the identification of OR-specific chaperone proteins which may allow

214 functional expression of ORs with cryptic mutations, further underscoring the high degree of sequence

215 diversification ORs are enabled to possess ${ }^{29,30}$. Using a diverse set of odorants, here we have

216 performed a functional in vivo characterization of the OR repertoire of Mus musculus. Linking the

217 activity of the receptor repertoire to an extensive set of molecular property descriptors parameterizing

218 the physical-chemical properties of the odorants, we learned that ORs displayed a continuum of tuning

219 breadths. Similarities between sparse sets of molecular properties could be used to predict receptor

220 response patterns. Finally, analyses linking odorant selectivity and amino acid residues most

221 consistently identified a series of poorly conserved residues located primarily in the upper half of the

222 transmembrane domains.

224 While our test odor panel, and resulting deorphanized receptor set, was broad in the coverage of odor

225 and receptor spaces, the data was by no means all-encompassing. 20 PCs were required to cover $90 \%$

226 of variance in the 52 set of tested odorants, whereas the full set of 4732 small molecules required 74

227 PCs to achieve comparable coverage (Figure 1 - Supplementary figure 1B). Our analyses therefore

228 likely reflect lower bound estimates of chemical and receptor response spaces. Nevertheless, these

229 results also imply that a substantial amount of the information in the molecular property descriptors is

230 highly redundant. Furthermore, we note that the dimensionality of the tested odorants in receptor

231 response space is higher than the dimensionality of the odorants in chemical space, with 34 PCs

232 needed to explain more than $90 \%$ of the response variance (Figure 1 - Supplementary figure 1B). This 
233 increased dimensionality indicates there are facets of odor response by ORs that are poorly explained

234 by a similar number of flat surfaces in chemical space described by molecular property descriptors.

235 Although the molecular property descriptors used in this study can explain and predict response

236 similarities, further searches for latent descriptors capable of better associating odor properties to

237 their receptor responses may improve these associations ${ }^{31,32}$.

239 Several implications arise from our observation that diverse odorants share commonalities that relate

240 their receptor responses to amino acid residues. First, the poor conservation of positions harboring

241 residues exhibiting predictive power and convergent evolution suggest a mechanism by which flexible

242 chemical recognition can be achieved by a family of proteins while maintaining a degree of

243 conservation necessary for functional protein integrity and activation of conserved downstream

244 signaling cascades. Second, the association of the third, fifth, and sixth transmembrane domains with

245 odor selectivity are also consistent with site-directed mutagenesis efforts on single ORs that have been

246 shown to influence OR responses ${ }^{33-37}$. Finally, these results are consistent with recent evidence from

247 structural elucidation of an ionotropic insect OR, which revealed a single binding pocket for a

248 structurally diverse odorants ${ }^{38}$. Future structural elucidation of mammalian ORs will enable direct

249 addressing of the modes of odorant-OR interactions.

251 In summary, we have provided a systematic, quantitative analysis of the primary representation of an

252 odor, as registered by the differential responses of individual ORs. Our results and analyses provide a

253 foundational framework for investigating how these primary odorant representations are transformed

254 into subsequent representations to ultimately guide behavioral outputs. 
bioRxiv preprint doi: https://doi.org/10.1101/2021.12.27.474279; this version posted December 28, 2021. The copyright holder for this preprint (which was not certified by peer review) is the author/funder. All rights reserved. No reuse allowed without permission.

\section{Supplementary figures:}

A

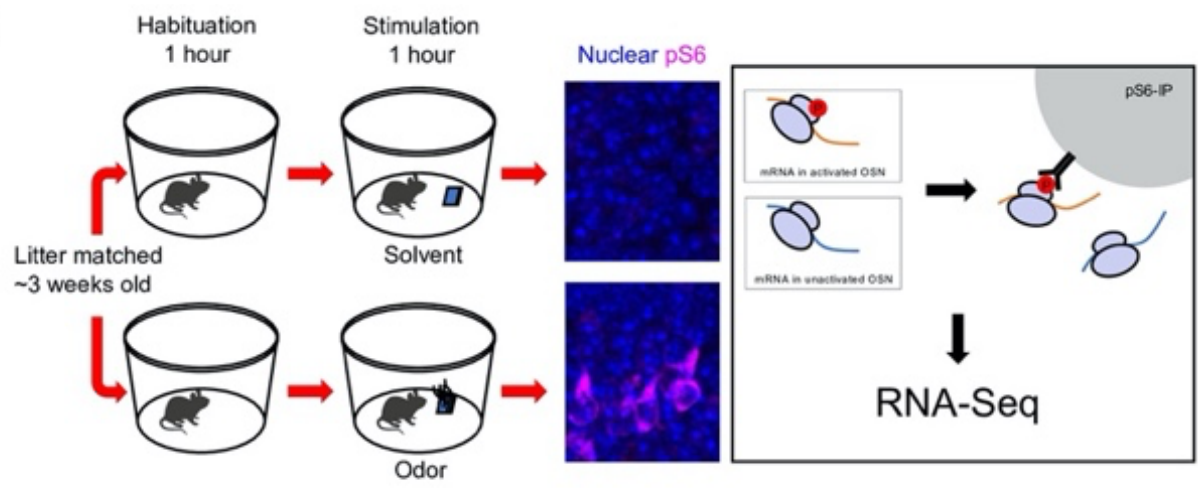

B
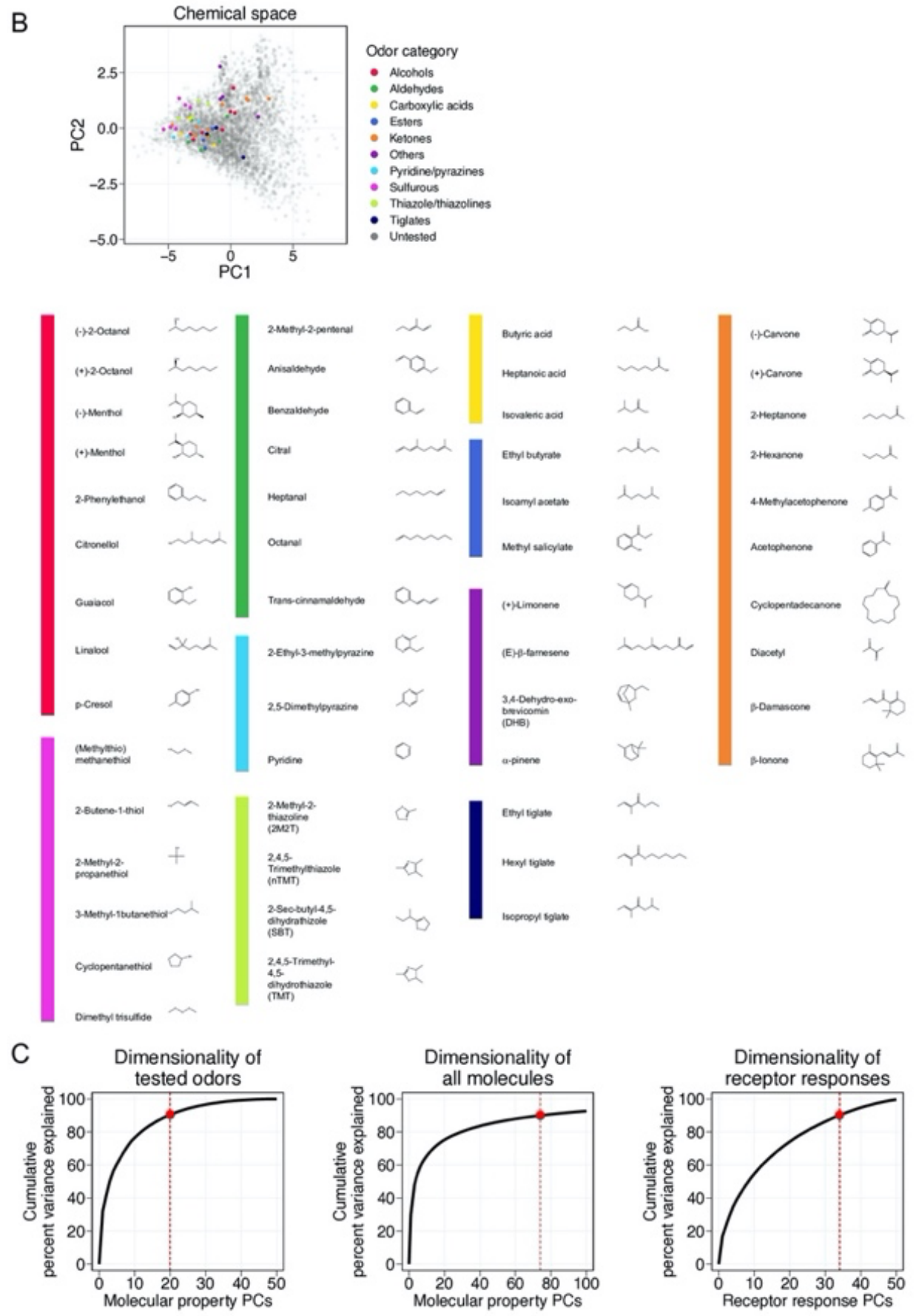
Supplementary figure 1. Details of chemical and receptor space sampling. A, Schematic of the pS6-IP-Seq experiment. Litter matched, 3 weeks old mice were used. Mice were first habituated to an odor-free environment for 1 hour. One mouse then received exposure to an odor stimulus, while another received exposure to the solvent, again for 1 hour. Whole olfactory mucosa was then harvested and immunoprecipitated using an antibody against pS6 and subjected to RNA-Seq. B, Top, tested odorants in chemical space colored by odor category. Bottom, molecular structures of tested odorants sorted by odor category. C, Left, the cumulative percent variance of the tested odorants in chemical space explained as a function of included PCs of molecular properties. A minimum of twenty PCs was required to capture at least $90 \%$ of the variance for the test odorants in chemical space. Middle, the cumulative percent variance of all molecules in chemical space explained as a function of included PCs of molecular properties. A minimum of seventy-four PCs was required to capture at least $90 \%$ of the variance for all molecules in chemical space. Right, the cumulative percent variance of the tested odorants in response space explained as a function of included PCs of receptor responses. A minimum of thirty-four PCs was required to capture at least $90 \%$ of the variance for the test odorants in response space. 

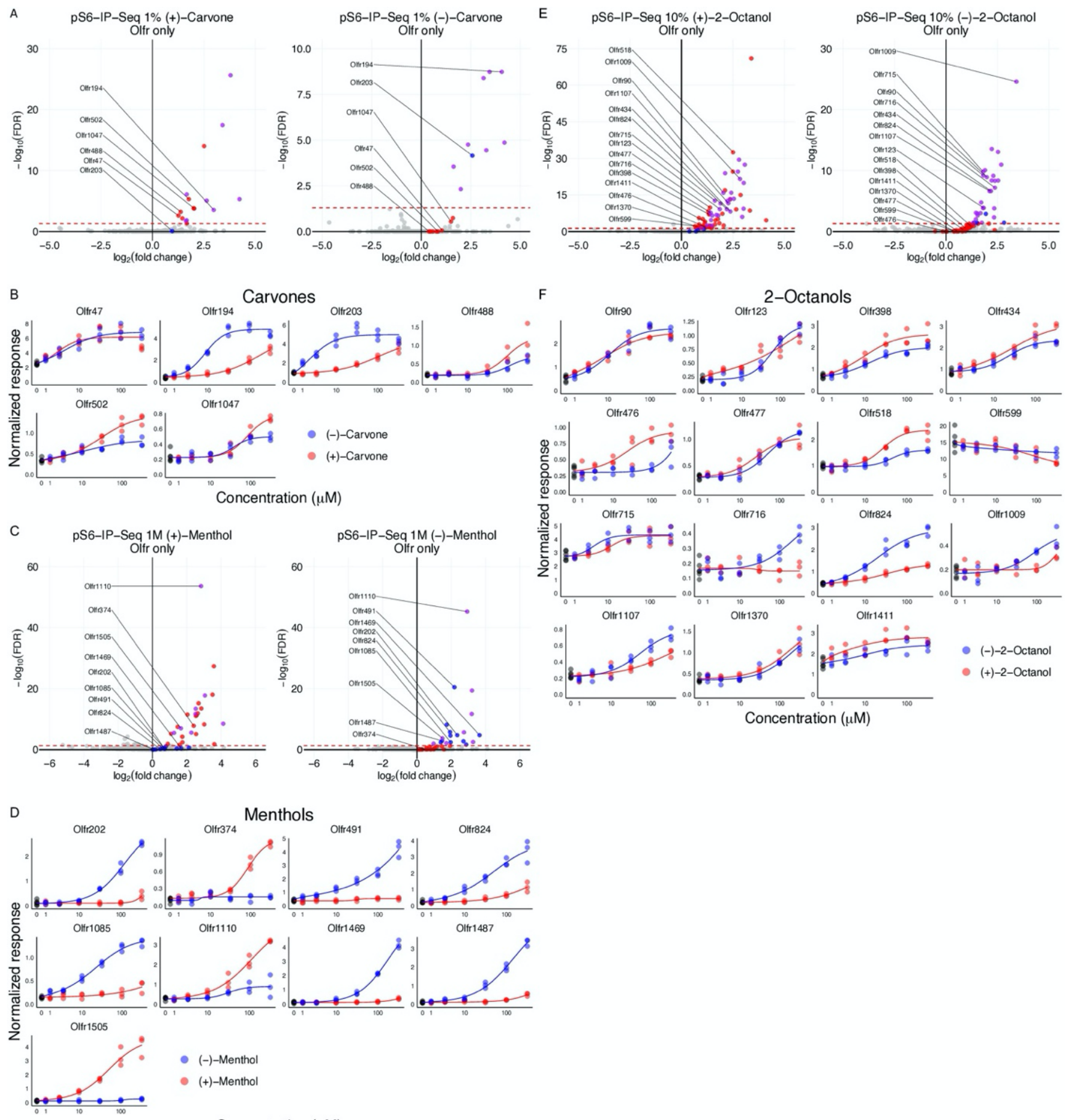

Concentration $(\mu \mathrm{M})$

Supplementary figure 2. Details of in vitro validation. A, Volcano plots for pS6-IP-Seq results by exposure of mice to $1 \%(+)$-carvone and 1\% (-)-carvone. A red line at FDR $=0.05$ is drawn. ORs enriched by just (+)-odorant are colored red while ORs enriched by just (-)-odorant are colored blue. ORs enriched by both enantiomers are colored purple. Labeled ORs were validated in vitro. B, Dose response curves of ORs displaying in vitro response to at least one of the tested carvone enantiomers. C, Volcano plots for pS6-IP-Seq results using 1M (+)-menthol and 1M (-)-menthol. D, Dose response curves of ORs displaying in vitro responses towards menthol enantiomers. E, Volcano plots for pS6-IP-Seq results using 10\% (+)-2-octanol and 10\% (-)-2-octanol. F, Dose response curves of ORs displaying in vitro responses towards 2-octanol enantiomers. 


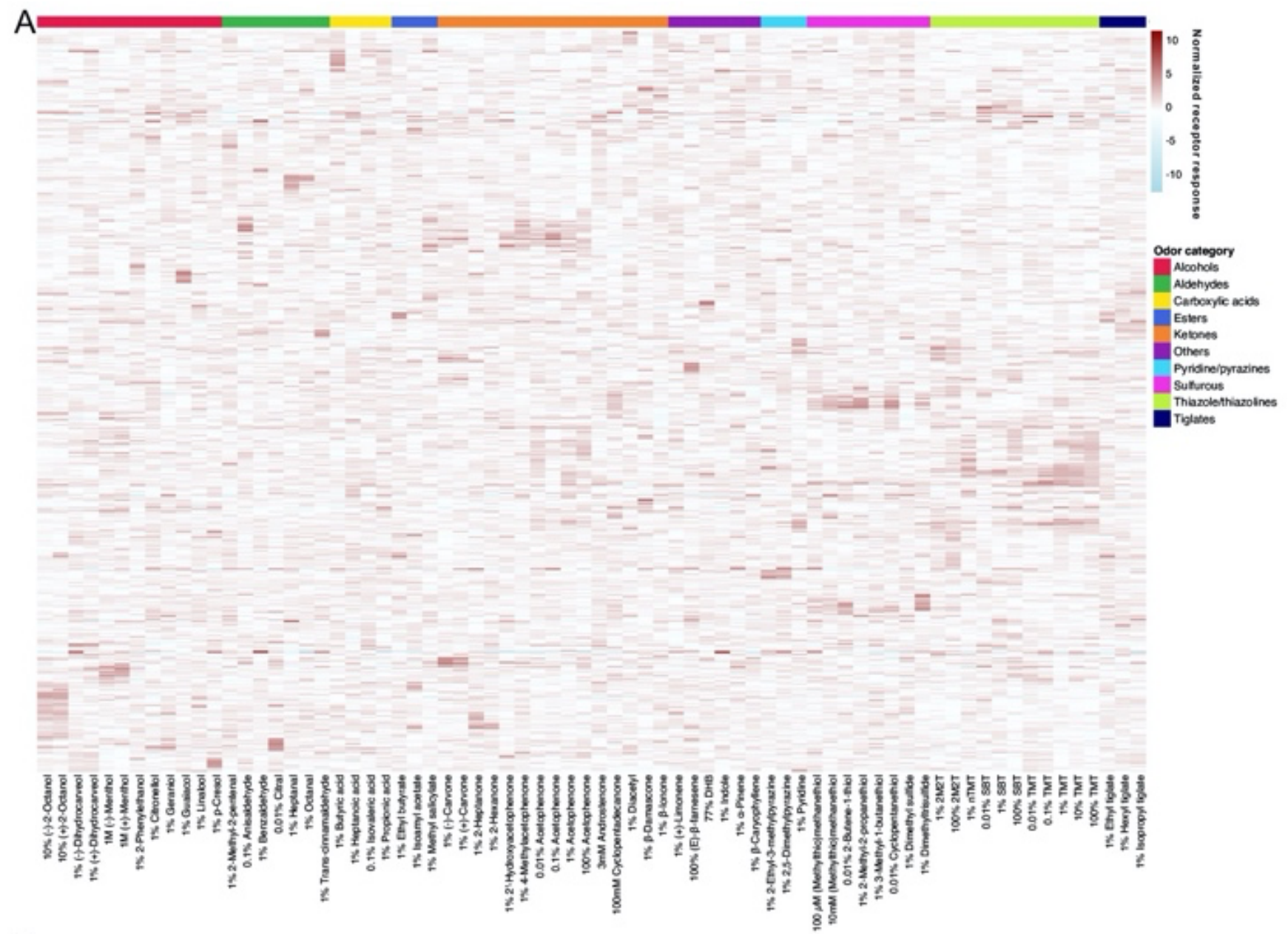

B Odor response distribution

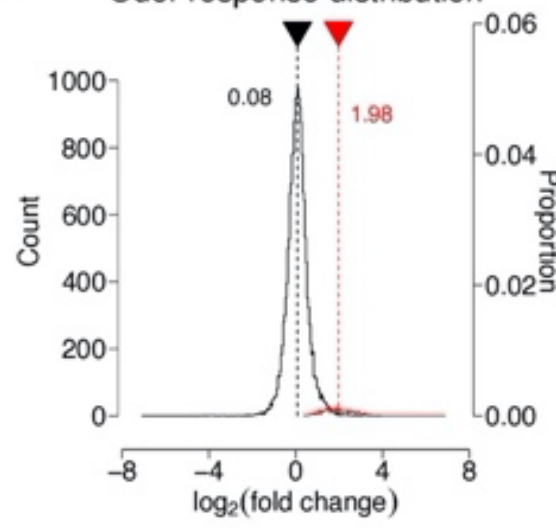

Odor response distribution
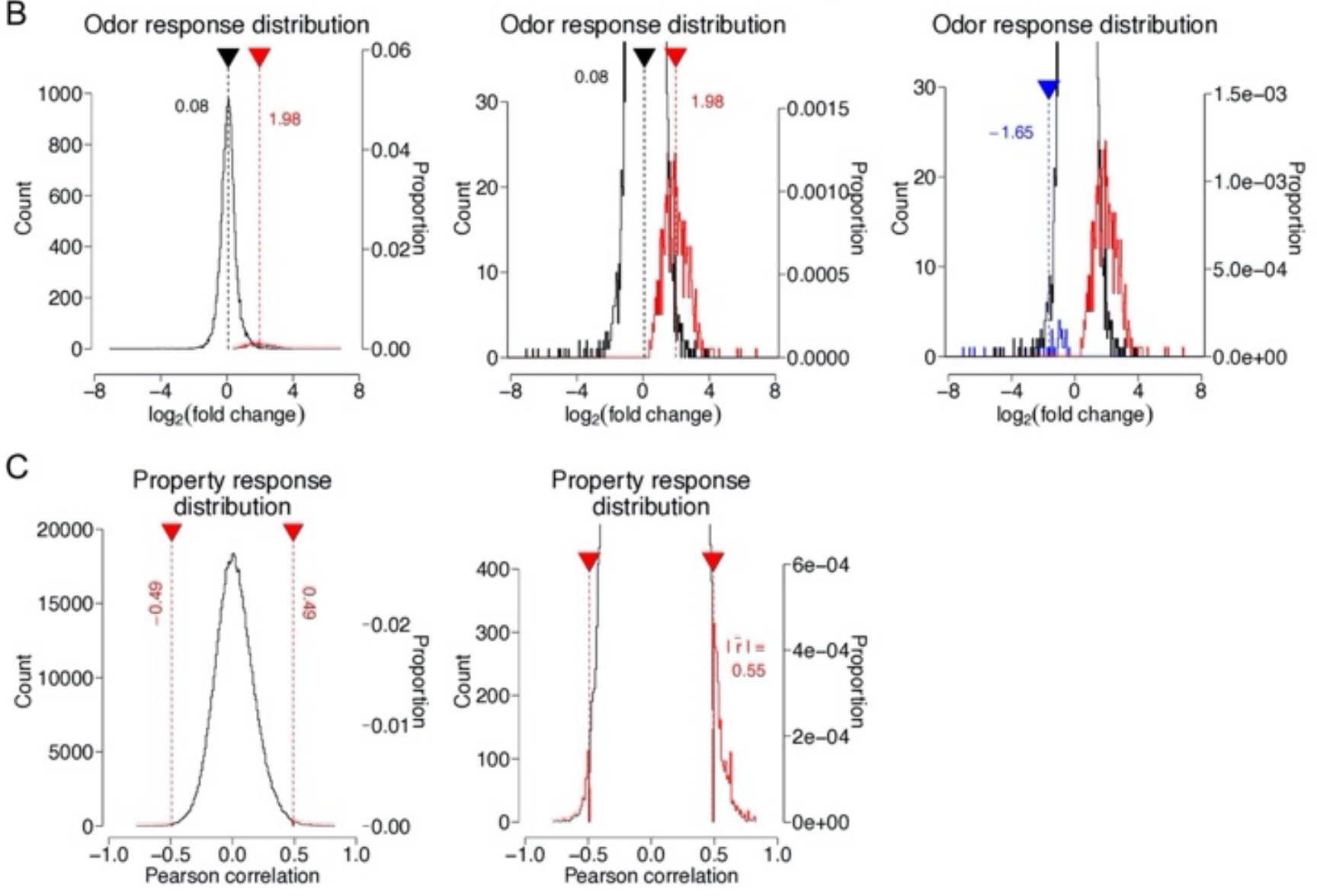
Supplementary figure 3. Details of OR response properties. A, Odorant responses determined by pS6-IP-Seq across all tested 72 odorants/concentrations visualized by heatmap. A total of 555 ORs are determined to respond to at least one of these odorants/concentrations. Responses are z-scored by odor. The following odorants are abbreviated: 2-methyl-2-thaizoline (2M2T), 2,4,5-trimethyl-4,5dihydrothiazole (TMT), 2-sec-butyl-4,5-dihydrothiazole (SBT), 2,4,5-trimethylthiazole (nTMT), and 3,4-dehydro-exo-brevicomin (DHB). Odorants are sorted by functional group while ORs are sorted using correlation distance. B, Left, histogram (bin size $=0.05$ ) of the distributions of OR responses across the panel of 52 odorants tested at low concentrations. Significant OR-odor pairs $\left(\log _{2} \mathrm{FC}>0\right.$ and FDR $<0.05$ ) are colored red, while non-significant pairs are colored black. OR-odor pairs that were classified as nonsignificant had an average $\log _{2} \mathrm{FC}$ enrichment of 0.08 by pS6-IP-Seq. OR-odor pairs classified as significant had an average $\log _{2} \mathrm{FC}$ enrichment of 1.98 by pS6-IP-Seq (nonsignificant responses $n=18808$, significant responses $n=692$ ). Middle, zoomed in. Right, A small number of inhibitory responses $\left(\log _{2} F C<0\right.$ and FDR $\left.<0.05\right)$ were observed in the data. These responses were otherwise classified as nonsignificant $(n=44)$. C, Left, distribution of the OR-molecular property pairwise Pearson correlation coefficients using the 52 odorants tested at low concentrations (bin size $=0.01$ ). At an FDR $<0.05$, the Pearson correlation coefficient cutoffs were -0.49 and 0.49 for negative and positive correlations respectively, with an absolute average of 0.55 for significant correlations (nonsignificant correlations $n=676158$, significant correlations $n=2967$ ). Right, zoomed in. 
A

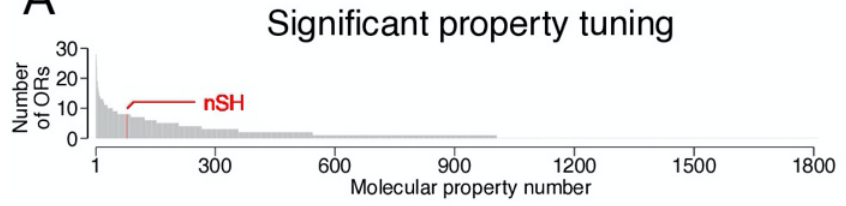

B

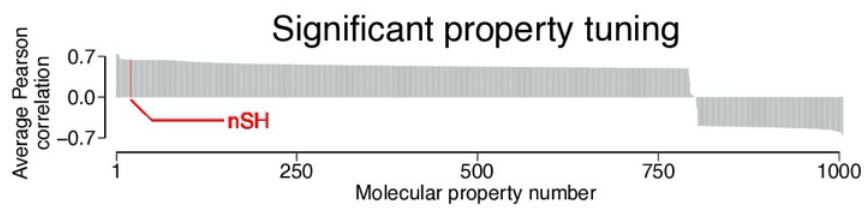

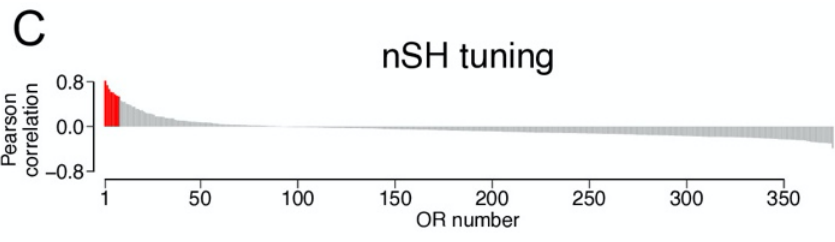

nSH tuning

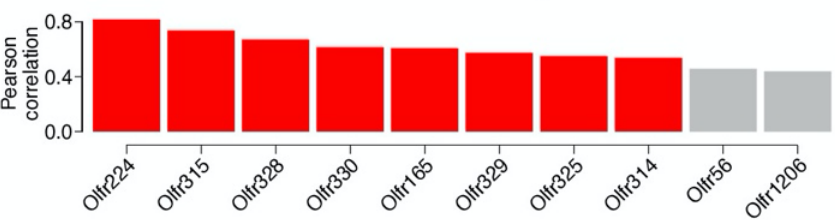

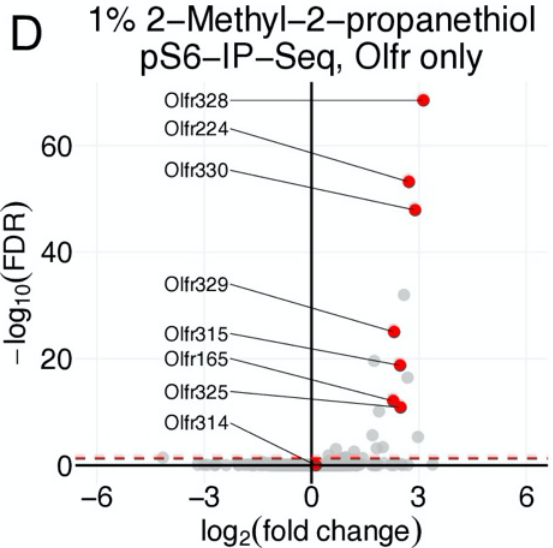

1\% 3-Methyl-1-butanethiol pS6-IP-Seq, Olfr only

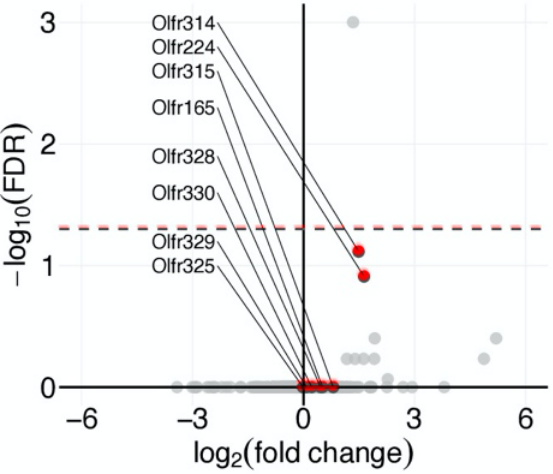

0.01\% Cyclopentanethiol pS6-IP-Seq, Olfr only

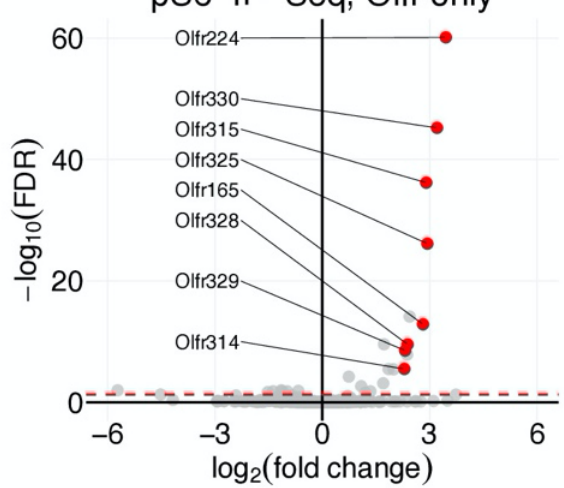

$100 \mu \mathrm{M}$ (Methylthio)methanethiol pS6-IP-Seq, Olfr only

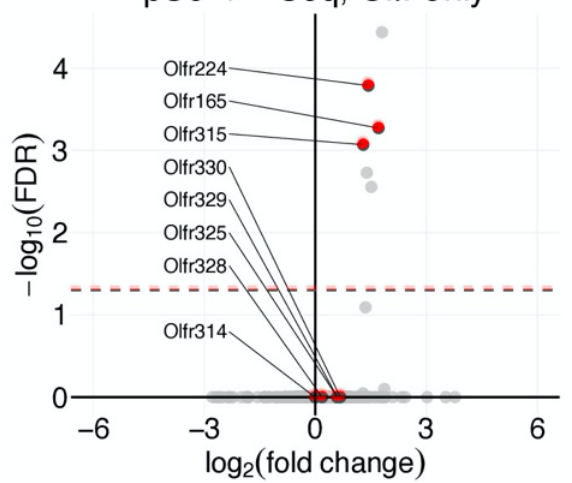

0.01\% 2-Butene-1-thiol pS6-IP-Seq, Olfr only
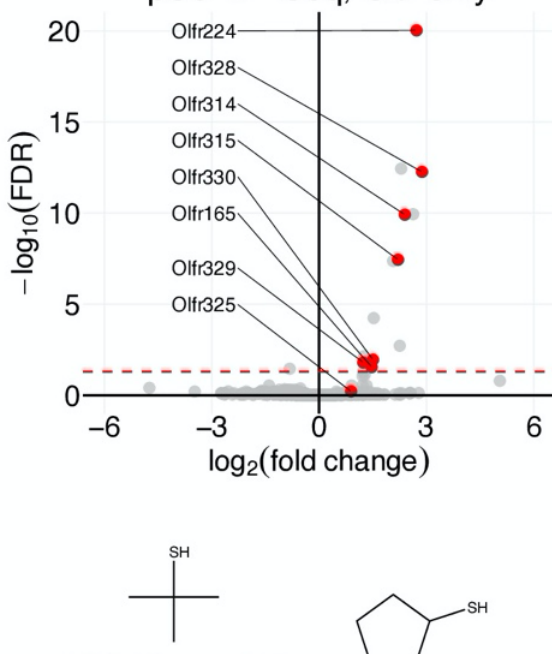

2-Methyl-2-propanethiol

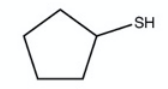

Cyclopentanethiol
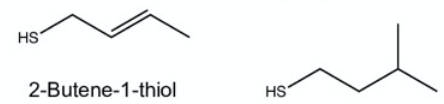

$\succ_{\mathrm{S} H}$

3-Methyl-1-butanethiol

(Methylthio)methanethiol

Supplementary figure 4. Details of thiol property tuning. A, Tuning towards molecular properties described by the number of significant OR-molecular property associations. Out of the 1811 molecular properties, 1005 were tuned towards by at least one OR. Molecular property nSH (number of thiol groups) is highlighted in red. B, Visualization of the average Pearson correlation tuning value for ORs that were significantly tuned toward each of the 1005 molecular properties. C, Top, tuning of individual ORs, measured by Pearson correlation, towards $\mathrm{nSH}$. Bottom, the top ten ORs displaying $\mathrm{nSH}$ tuning. Eight of these ten ORs were significant (FDR < 0.05). D, Raw pS6-IP-Seq differential expression data, visualized by volcano plot, with chemical structures of the tested thiol odorants. ORs that were tuned towards thiol are highlighted in red. A consensus of thiol odorant response can be observed for the ORs tuned towards $\mathrm{nSH}$. 
A
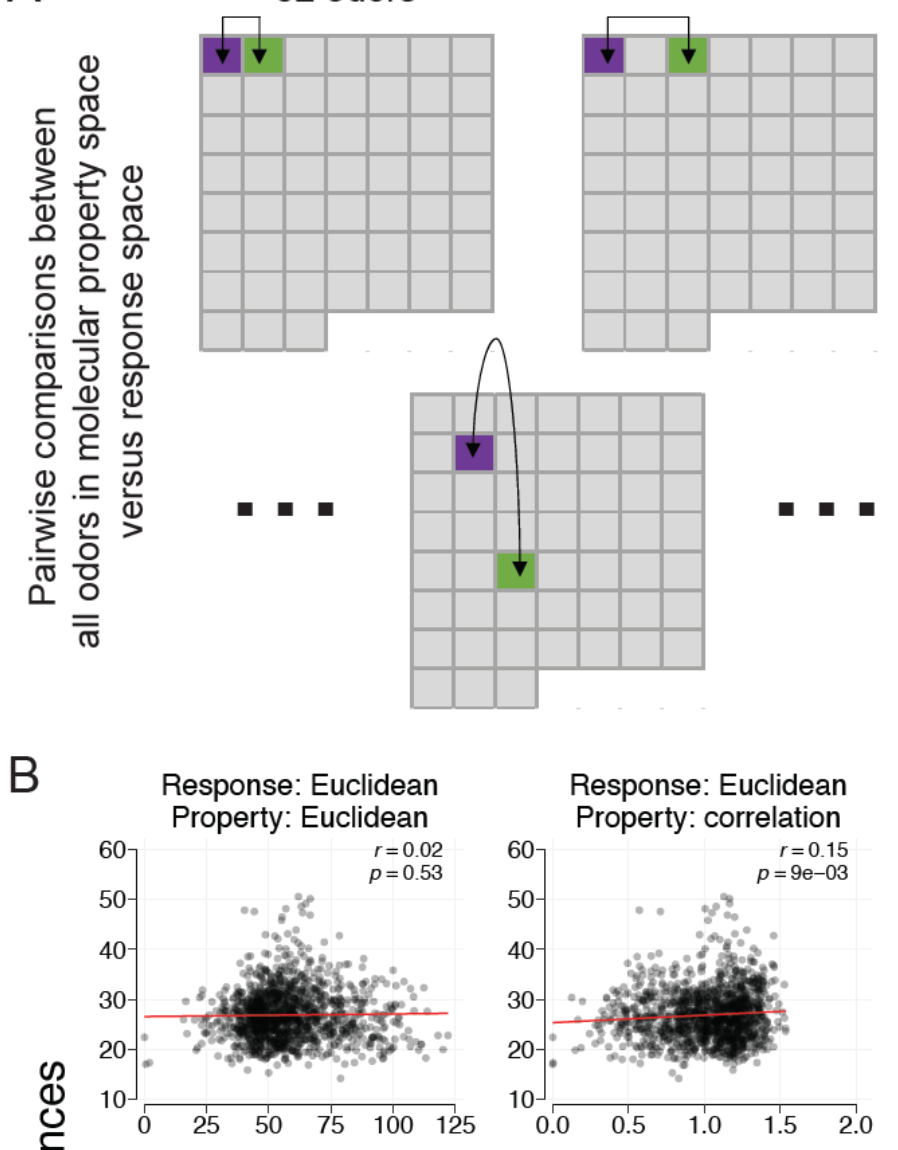

Response: correlation Property: Euclidean
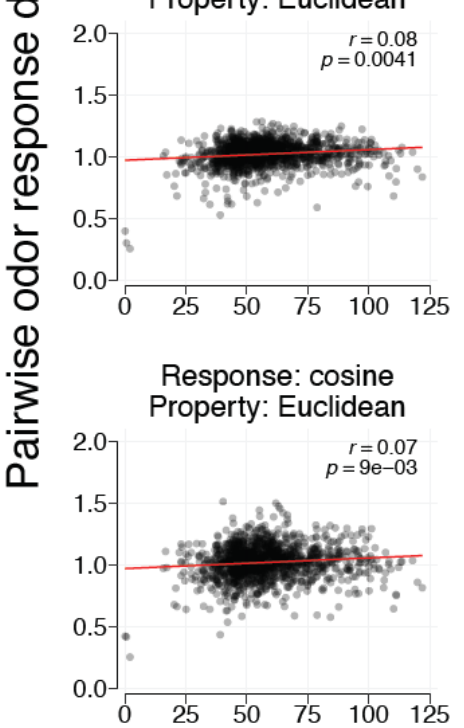
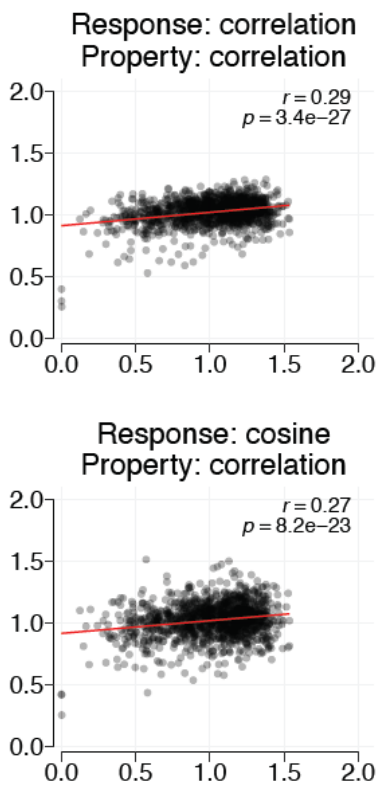
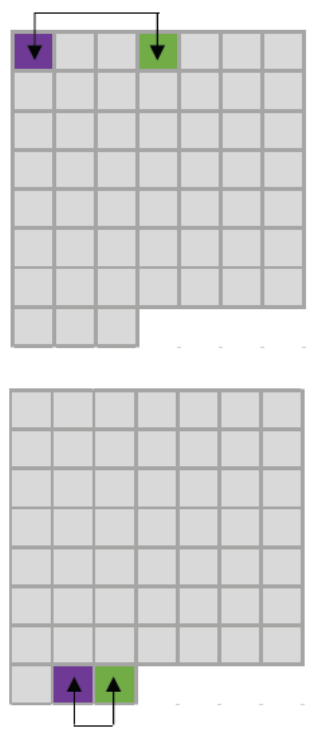

Response: Euclidean

Property: cosine

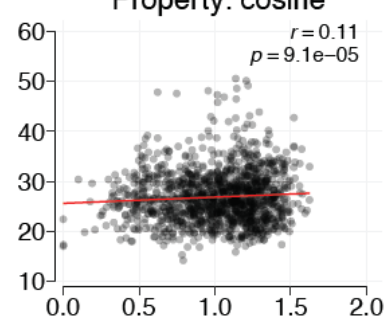

Response: correlation Property: cosine

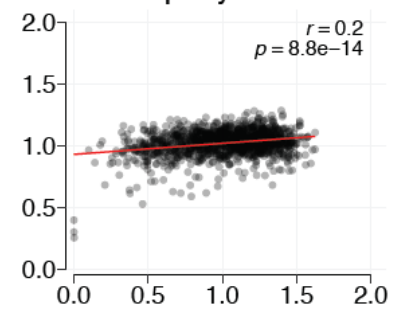

Response: cosine Property: cosine

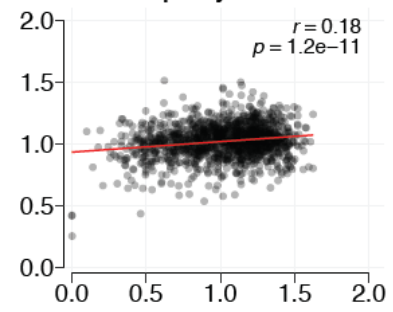

Pairwise odor molecular properties distances

Supplementary figure 5 . Details of the pairwise comparisons between odorants in response and molecular property space. A, Schematic of how pairwise distance comparisons between odorants were calculated for figures 3 A and 3 C. B, Correspondence between odorants in response and molecular property spaces. Three (Euclidean, correlation, and cosine) different distance metrics were used. Pearson correlation coefficients and $p$-values are reported for each combination of distance metric. 

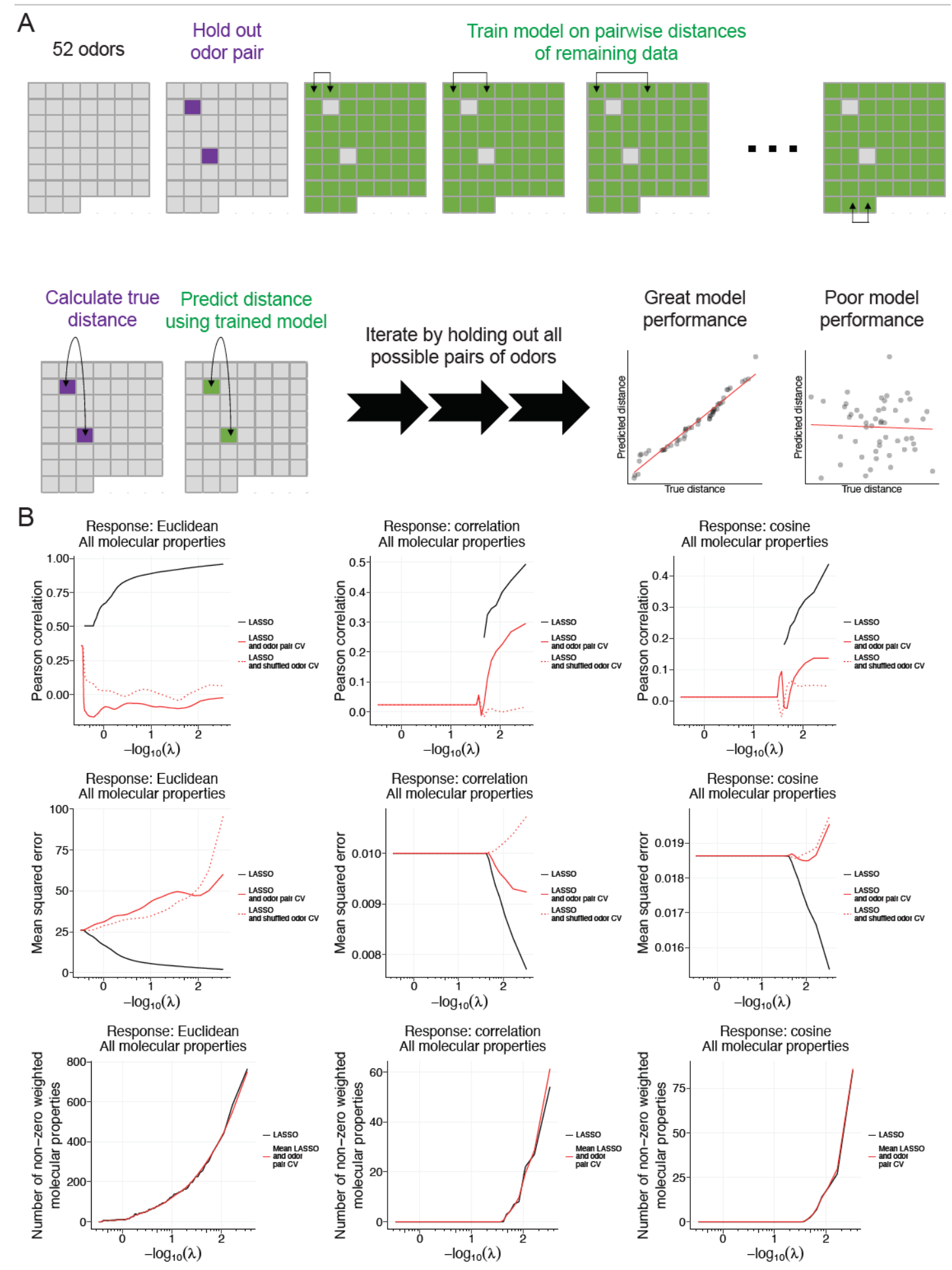

Supplementary figure 6. Details of the odor pair cross-validation for regression models. A, Schematic of how odor pair cross-validation was performed. Pairs of odorants were iteratively held-out from training. Distances between held-out odorants were iteratively predicted and regressed against true distances to report a Pearson correlation and mean squared error. B, Results of LASSO regression using various metrics to quantify distances between odorants in response space. Molecular property distances were quantified by Euclidean distances. Reported are Pearson correlation coefficients, mean squared error, and the number of non-zero weighted molecular properties as a function of varying the loss function $(\lambda)$. 


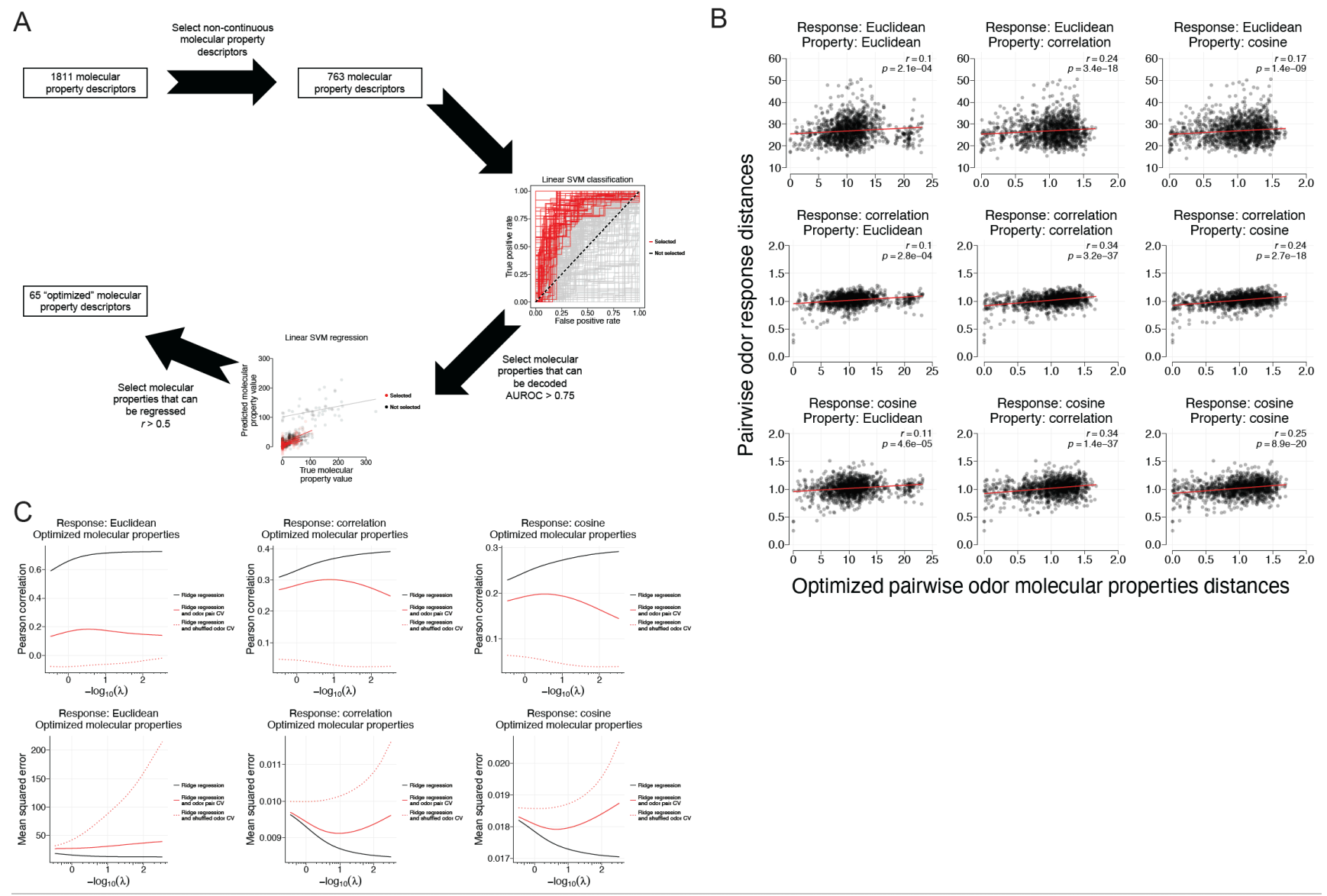

\section{Supplementary figure 7. Performance of optimized molecular properties in predicting receptor} response similarities. A, Schematic of how "optimized" molecular properties were selected. First, a set of 763 non-continuous descriptors were subset. Molecular properties that could be linearly decoded and regressed (with cross-validation) from the OR responses were then further selected using classifier AUROC thresholds of 0.75 and regressor $r$ thresholds of 0.5 . Ultimately 65 molecular properties passed these criteria and were considered "optimized". B, Correspondence between odorants in response and "optimized" molecular property spaces. Euclidean, correlation, and cosine distance metrics are used to report Pearson correlation coefficients and $p$-values. C, Results of ridge regression using various metrics to quantify distances between odorants in response space. Generalizability was evaluated by odor pair cross-validation. Reported are Pearson correlation coefficients and mean squared error as a function of varying the loss function $(\lambda)$. 
bioRxiv preprint doi: https://doi.org/10.1101/2021.12.27.474279; this version posted December 28,2021 . The copyright holder for this preprint (which was not certified by peer review) is the author/funder. All rights reserved. No reuse allowed without permission.

A
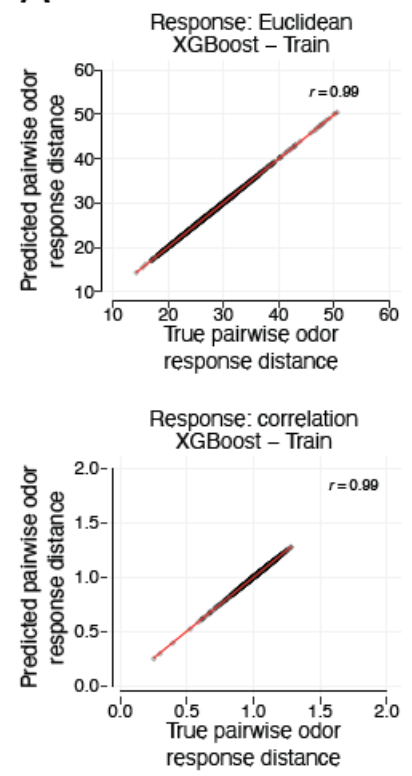

Response: cosine

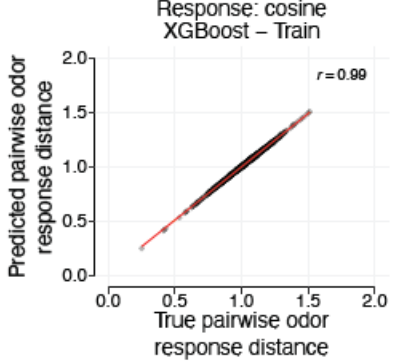

B

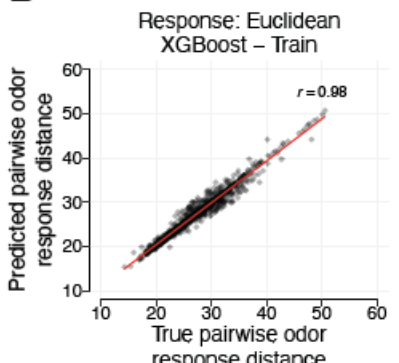

response distance

Response: correlation

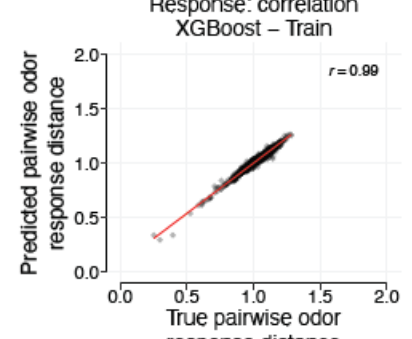

response distance

Response: cosine

XGBoost - Train

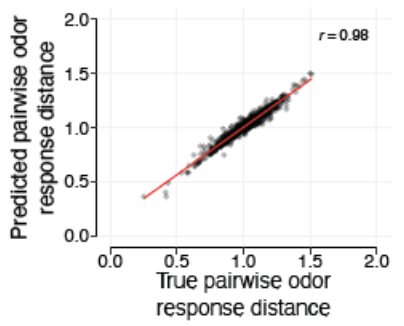

All molecular properties
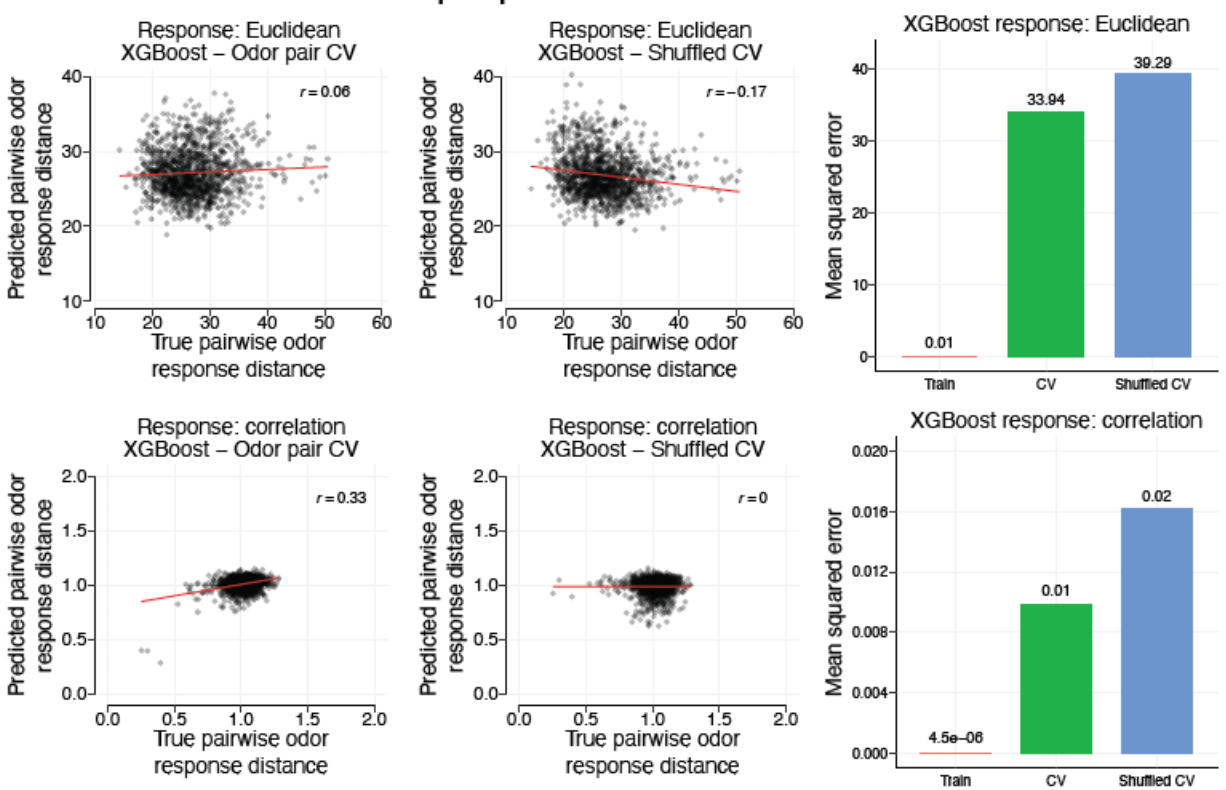

Response: cosine
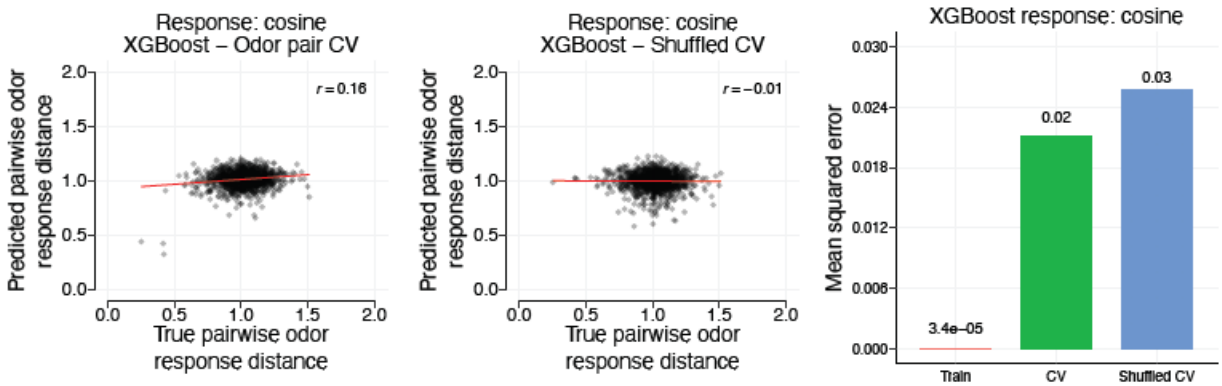

\section{Optimized molecular properties}
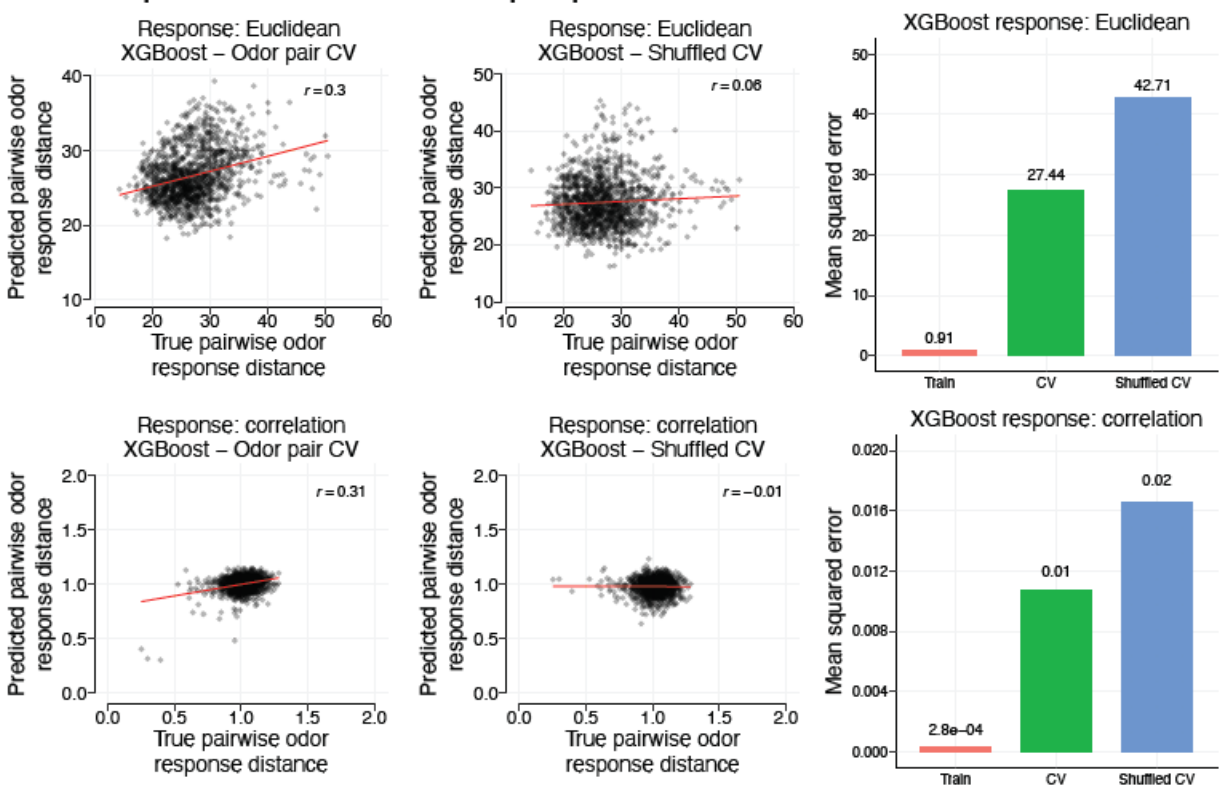

Response: cosine
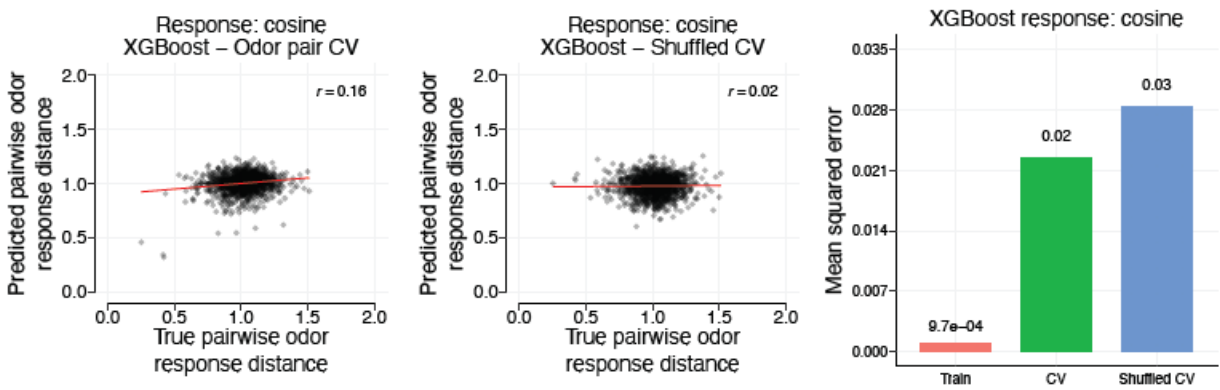
Supplementary figure 8. Results of using the XGBoost model framework. A, Using default XGBoost hyperparameters with 1811 molecular property descriptors, we asked how well does odor molecular property similarity predict receptor response similarity. Response similarities were calculated using Euclidean, correlation, and cosine distances. Odor pair cross-validation was performed to evaluate the generalizability of the models. B, Results of the XGBoost models when only the 65 set of "optimized" odor molecular properties were used as predictors and odor pair cross-validation is performed. The positive predictive abilities of these sparse 65 odor molecular properties, independent of the distance metric used) demonstrates odor similarity in response space can be approximated with parsimonious combinations of odor molecular properties. 


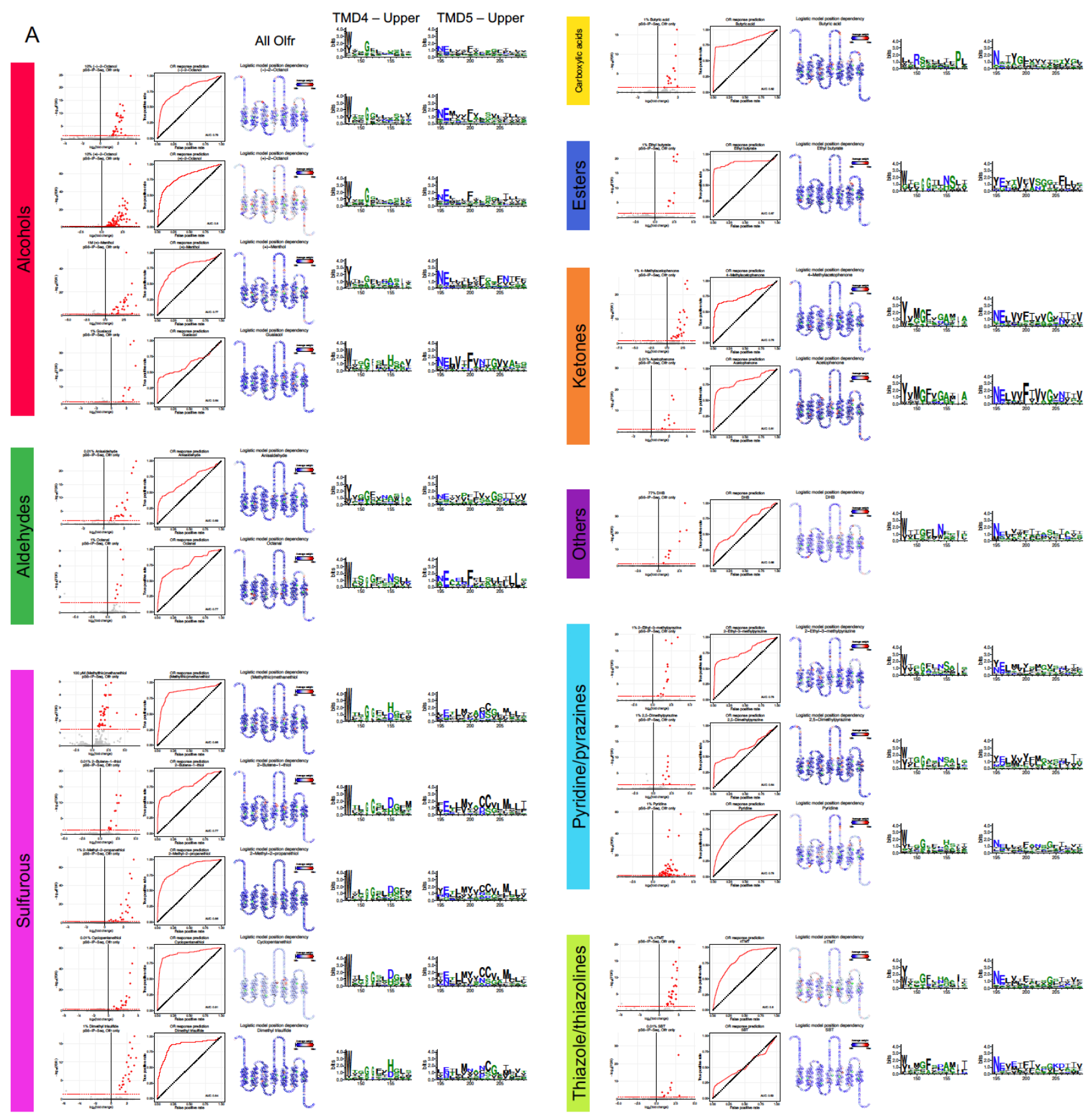

Supplementary figure 9. Details of logistic regression to uncover sequence-function relationships of ORs. A, Data for odorants solvable by regularized logistic regression (AUROC $>0.5$ ) is shown. For each odor, shown is a volcano plot highlighting responsive ORs in red $\left(\log _{2} \mathrm{FC}>0\right.$ and FDR $\left.<0.05\right)$ and non-responsive ORs in gray. Aligned amino acid sequences were used as inputs to predict response likelihoods of a held-out $10 \%$ of ORs for 100 repetitions. The receiver operating characteristic (ROC) curve for held-out data is shown. Positions harboring amino acids assigned non-zero weights were averaged and visualized by snakeplot. Ballesteros-Weinstein $\mathrm{x} .50$ numbers for each TMD are highlighted in green. The consistency of high weights assigned to residues localized to the upper halves of the fourth and fifth transmembrane domains motivated visualization of the amino acid distributions of responsive ORs by WebLogo. An enrichment of cysteine residues can be seen amongst ORs responsive to sulfurous odorants at positions 202 and 203 in TMD5. Similarly, an enrichment of methionine residues can be seen amongst ORs responsive to sulfurous odorants at positions 199 and 206. 

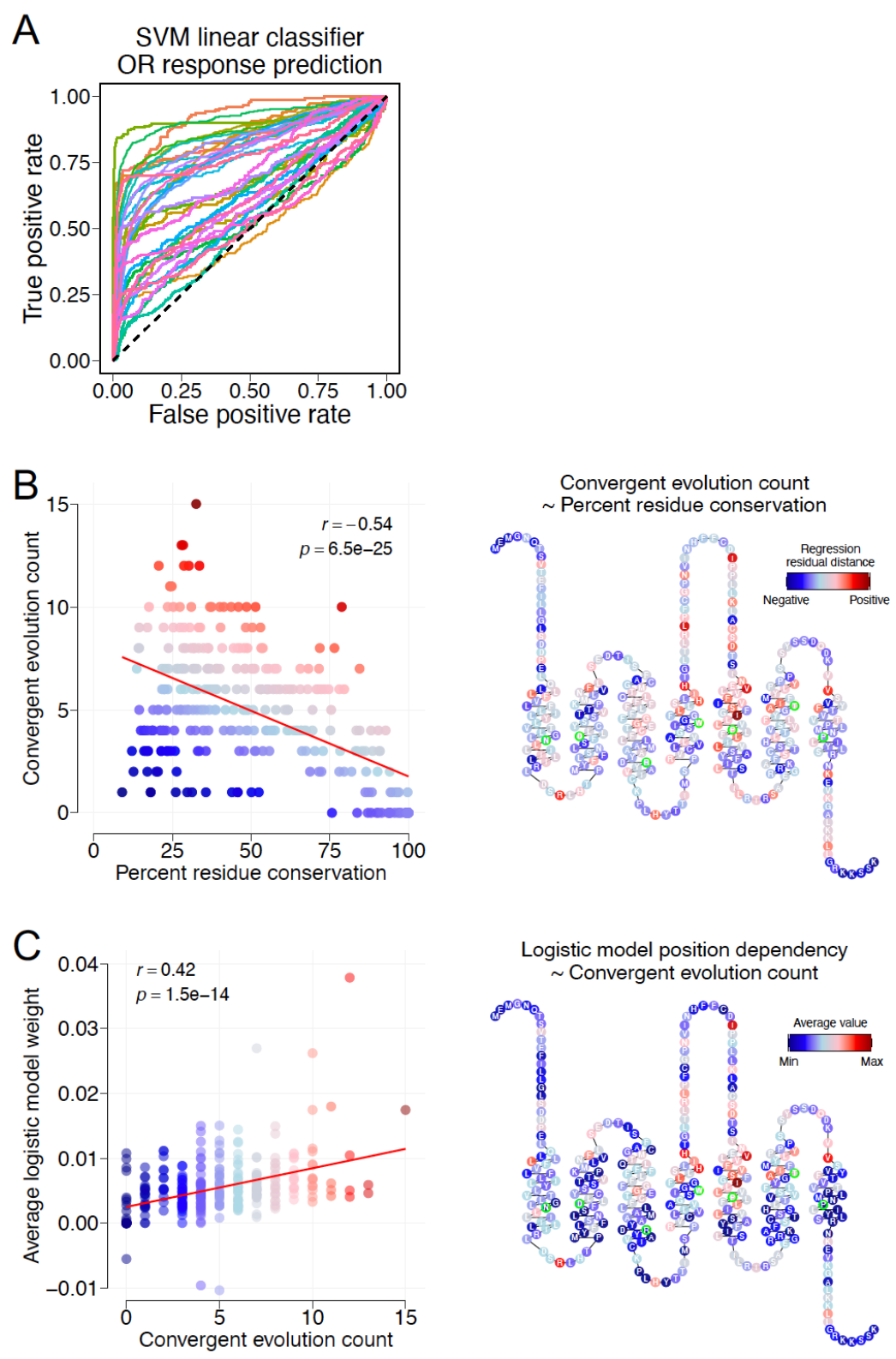

\section{Supplementary figure 10. Details of SVM classifiers and comparing between approaches. A,} Results of using a linear SVM classifier to predict OR response likelihoods across 100 repetitions of splitting ORs into $90 \%$ training and $10 \%$ testing sets. Models were trained and optimized using ten-fold cross-validation. Prediction likelihoods across 100 repetitions were compounded to generate single ROC curves for single odorants. AUROC values for each odorant are reported in supplementary table 4. B, Regressing convergent evolution counts by percent residue conservation reveals an anti-correlation ( $r=-0.54, p=6.5 \mathrm{E}-25)$. Snakeplot is colored by the distance of each data point from the line of regression. Ballesteros-Weinstein x.50 numbers for each TMD are highlighted in green. C, Regressing logistic model dependency, evaluated by average positional weight, by convergent evolution count reveals consistency between approaches $(r=0.42, p=1.5 \mathrm{E}-14)$. 


\section{Methods:}

\section{Phosphorylated S6 ribosomal capture (pS6-IP)}

268 Mice used for pS6-IP were 3 weeks old, mixed sex, and littermates. Mice were killed by

$269 \mathrm{CO}_{2}$ asphyxiation and cervical dislocation. Olfactory tissue was rapidly dissected in Buffer B (2.5 mM

270 HEPES KOH pH 7.4, 0.63\% glucose, $100 \mu \mathrm{g} / \mathrm{mL}$ cycloheximide, $5 \mathrm{mM}$ sodium fluoride, $1 \mathrm{mM}$ sodium

271 orthovanadate, $1 \mathrm{mM}$ sodium pyrophosphate, $1 \mathrm{mM} \beta$-glycerophosphate, in Hank's balanced salt

272 solution). Tissue pieces were then minced in $1.35 \mathrm{~mL}$ Buffer $\mathrm{C}(150 \mathrm{mM} \mathrm{KCl}, 5 \mathrm{mM} \mathrm{MgCl}, 10 \mathrm{mM}$

273 HEPES KOH pH 7.4, $0.100 \mu \mathrm{M}$ Calyculin A, 2 mM DTT, 100 U/mL RNAsin, $100 \mu \mathrm{g} / \mathrm{mL}$ cycloheximide,

274 protease inhibitor cocktail, $5 \mathrm{mM}$ sodium fluoride, $1 \mathrm{mM}$ sodium orthovanadate, $1 \mathrm{mM}$ sodium

275 pyrophosphate, $1 \mathrm{mM} \beta$-glycerophosphate)and subsequently transferred to homogenization tubes for

276 steady homogenization at $250 \mathrm{rpm}$ three times and at $750 \mathrm{rpm}$ nine times at $4{ }^{\circ} \mathrm{C}$. Samples were then

277 transferred to a $1.5 \mathrm{~mL}$ LoBind tube (Eppendorf 022431021) and clarified at 2000xg for $10 \mathrm{~min}$ at $4{ }^{\circ} \mathrm{C}$.

278 The low-speed supernatant was transferred to a new tube on ice, and $90 \mu \mathrm{L}$ of NP40 (Sigma

$27911332473001)$ and $90 \mu \mathrm{L}$ of 1,2-diheptanoyl-sn-glycero-3-phosphocholine (DHPC, Avanti Polar Lipids

$280850306 \mathrm{P}, 100 \mathrm{mg} / 0.69 \mathrm{~mL}$ ) were added to this solution. This solution was mixed and then clarified at a

281 max speed $(17,000 \mathrm{xg})$ for $10 \mathrm{~min}$ at $4{ }^{\circ} \mathrm{C}$. The resulting high-speed supernatant was transferred to a

282 new tube where $20 \mu \mathrm{L}$ was saved and transferred to a tube containing $350 \mu \mathrm{L}$ buffer RLT. To the

283 remainder of the sample, $1.3 \mu \mathrm{L}$ of $100 \mu \mathrm{g} / \mathrm{mL}$ cycloheximide, $27 \mu \mathrm{L}$ of phosphatase inhibitor cocktail

284 (250 mM sodium fluoride, $50 \mathrm{mM}$ sodium orthovanadate, $50 \mathrm{mM}$ sodium pyrophosphate, $50 \mathrm{mM} \beta$ -

285 glycerophosphate) and $6 \mu \mathrm{L}$ of anti-pS6 antibody (Cell Signaling D68F8) were added. The sample was

286 gently rotated for $90 \mathrm{~min}$ at $4{ }^{\circ} \mathrm{C}$. To prepare beads, $100 \mu \mathrm{L}$ of beads (Invitrogen 10002D) was washed

287 three times with $900 \mu \mathrm{L}$ of buffer $\mathrm{A}(150 \mathrm{mM} \mathrm{KCl}, 5 \mathrm{mM} \mathrm{MgCl}$, $10 \mathrm{mM} \mathrm{HEPES} \mathrm{KOH} \mathrm{pH} \mathrm{7.4,} \mathrm{10 \%} \mathrm{NP40,}$

$28810 \%$ BSA), and once with $500 \mu \mathrm{L}$ of buffer C. Sample homogenate was added to the beads and

289 incubated with gentle rotation for $60 \mathrm{~min}$ at $4{ }^{\circ} \mathrm{C}$. Following incubation, beads were washed with four 
290 times with $700 \mu \mathrm{L}$ of buffer D (350 mM KCl, 5 mM MgCl 2,10 mM HEPES KOH pH 7.4, 10\% NP40, 2 mM

291 DTT, $100 \mathrm{U} / \mathrm{mL}$ RNAsin, $100 \mu \mathrm{g} / \mathrm{mL}$ cycloheximide, $5 \mathrm{mM}$ sodium fluoride, $1 \mathrm{mM}$ sodium

292 orthovanadate, $1 \mathrm{mM}$ sodium pyrophosphate, $1 \mathrm{mM} \beta$-glycerophosphate). During the final wash,

293 beads were moved to room temperature, wash buffer was removed, and $350 \mathrm{~mL}$ of buffer RLT was

294 added. Beads were incubated in buffer RLT for 5 min at room temperature. Buffer RLT containing

295 immunoprecipitated RNA was then eluted and stored at $-80^{\circ} \mathrm{C}$ until clean up using a kit (Qiagen

296 74004). cDNA was generated using 11 rounds of amplification with 10 ng RNA input. DNA libraries

297 were prepared using a half-sized Nexterra XT DNA Library Preparation Kit (Illumina 15032354) protocol

298 as per the manufacturer's guidelines. Libraries were sequenced on either HiSeq 2000/2500 (50 base

299 pair single read mode) or NextSeq 500 (75 base pair single read mode) with 6-12 pooled indexed

300 libraries per lane.

RNA-Seq alignment, quantification, and differential expression analysis

303 Reads were aligned against a modified GRCm38.p6 (M25) reference, in which we deleted

ENSMUSG00000116179 (Olfr290), using STAR ${ }^{39}$ with --outFilterMultimapNmax 10. Reads mapping to

Olfr290 were inferred from ENSMUSG00000070459, with the rationale that this gene model included

ENSMUSG00000116179 plus untranslated regions. Gene-level read quantification was done using

$307 \mathrm{RSEM}^{40}$. Differential expression analysis was performed against all genes using Edge ${ }^{41}$. Gene

308 nomenclature was retrieved from BioMart ${ }^{42}$. Intact Olfr genes with identifiable sequences were

309 filtered, and p-values were then re-corrected by FDR. Only ORs exhibiting odor response to at least one

310 of the tested odorants $\left(\log _{2} \mathrm{FC}>0\right.$ and FDR $\left.<0.05\right)$ were considered. A total of 555 ORs responded

311 across the 72 different odorants at various concentrations. A total of 375 ORs were responsive to

312 unique odorants at the lowest tested concentrations. Raw and processed RNA-Seq datasets generated

313 as part of this study are available from NCBI GEO at accession GSE185415. 


\section{Source of odorants}

316 The following odors/concentrations were used for comparing molecular properties to receptor

317 responses: 1\% 2-methyl-2-pentenal (Sigma 294667), 1\% trans-cinnamaldehyde (Sigma C80687), 1\% 2-

318 heptanone (Sigma 537683 ${ }^{15}$ ), 1\% linalool (Sigma L2602), 1\% ethyl butyrate (Sigma W242713), 1\%

319 guaiacol (Sigma G10903), 1\% diacetyl (Sigma W237027), 1\% 2-ethyl-3-methylpyrazine (Sigma

320 W315508), 1\% 2,5-dimethylpyrazine (Sigma 17542015), 1\% benzaldehyde (Sigma W212717), 1\% (+)-

321 limonene (Sigma 183164), 1\% $\beta$-damascone (Sigma W324300), 1\% $\alpha$-pinene (Sigma W290267), 1\% 2-

322 methyl-2-thiazoline (Sigma M83406), 1\% citronellol (Sigma W230915), 1\% dimethyl trisulfide (Sigma

323 W327506), 1\% p-Cresol (Sigma C85751), 0.01\% citral (Sigma W230316), 1 M (+)-menthol (Sigma

324 224464), 1 M (-)-menthol (Sigma M2780), 0.01\% anisaldehyde (Sigma A88107), 1\% 4-

325 methylacetophenone (Sigma W267708), 1\% methyl salicylate (Sigma W274502), 1\% (+)-carvone (Sigma

326 22070), 1\% (-)-carvone (Sigma 22060), 1\% $\beta$-ionone (Sigma W259525), 1\% isopropyl tiglate (Sigma

327 W322903), 1\% hexyl tiglate (Sigma W500909), 1\% pyridine (Sigma 270970), 1\% butyric acid (Sigma

328 W222119), 0.01\% cyclopentanethiol (Sigma W326208), 0.01\% 2-butene-1-thiol (1717 CheMall Corp

329 OR116574), 100 mM cyclopentadecanone (Sigma C111201), 1\% 2-methyl-2-propanethiol (Sigma

330 109207), 0.01\% acetophenone (Sigma W200910), 0.1\% isovaleric acid (Sigma 129542), 1\% isoamyl

331 acetate (Sigma 306967), 1\% ethyl tiglate (Sigma W246000), 1\% heptanoic acid (Sigma W334812), 10\%

332 (+)-2-octanol (Sigma O4504), 10\% (-)-2-octanol (Sigma 147990), 1\% 2-hexanone (Sigma 103004), 1\% 2-

333 phenylethanol (Sigma 77861), 1\% 3-methyl-1-butanethiol (Sigma W385808), 1\% octanal (Sigma

334 O5608), 1\% heptanal (Sigma W254002), 1\% 2,4,5-trimethylthiazole (nTMT, Sigma 219185), 100\% (E)- $\beta$ -

335 Farnesene (Bedoukian P3500-9014), $100 \mu \mathrm{M}$ (methylthio)methanethiol (MTMT, synthesized ${ }^{15}$ ), 0.01\%

336 2-sec-butyl-4,5-dihydrothiazole (SBT, synthesized ${ }^{15}$ ), 77\% 3,4-dehydro-exo-brevicomin (DHB,

337 synthesized ${ }^{15}$ ), and 0.01\% 2,4,5-trimethyl-4,5-dihydrothiazole (TMT, synthesized ${ }^{14}$ ). 
339 For logistic regression and identifying residues with predictive power towards ligand selectivity,

340 odorants tested at the lowest concentration with at least 8 activated $\mathrm{ORs}\left(\log _{2} \mathrm{FC}>0\right.$ and FDR $\left.<0.05\right)$

341 were used to promote class stability. Thus, following odors were removed from consideration using

342 logistic regression compared to above: $100 \mathrm{mM}$ cyclopentadecanone, 1\% 2-heptanone, 1\% 2-

343 hexanone, 1\% 3-methyl-1-butanethiol, $1 \% \alpha$-pinene, $1 \%$ benzaldehyde, $1 \% \beta$-ionone, $1 \%$ ethyl tiglate,

$3441 \%$ heptanoic acid, $1 \%$ hexyl tiglate, $1 \%$ isopropyl tiglate, $1 \%$ linalool, $1 \%$ methyl salicylate, $1 \%(+)-$

345 limonene, $1 \%$ trans-cinnamaldehyde, and $0.1 \%$ isovaleric acid. The following odors were considered at 346 a modified concentration from above: 0.1\% TMT, 0.1\% acetophenone, and $10 \mathrm{mM} \mathrm{MTMT.}$

348 Odorants were excluded from all analysis if no ORs were identified as responsive at the tested

349 concentrations: $1 \% \beta$-Caryophyllene (Sigma W225207 ${ }^{15}$ ), 1\% dimethyl sulfide (Sigma 274380), 1\%

350 geraniol (Sigma W250716), 1\% indole (Sigma W259378), 1\% (-)-dihydrocarveol (Sigma 37278), 1\% (+)-

351 dihydrocarveol (Sigma 37277), 1\% propionic acid (Sigma 109797), 3mM androstenone (Sigma 284998),

352 or if the number of ORs identified as responsive were more than five standard deviations away from

353 the mean: 1\% 2'-hydroxyacetophenone (Sigma H18607).

\section{Chemical space estimation}

356 To estimate chemical space, we first identified 4680 small molecules commonly found in foods and

357 fragrances from http://www.thegoodscentscompany.com/ ${ }^{16}$. Three dimensional structures for these

358 molecules and the 52 in the test odor set were then downloaded from PubChem, and 5666 molecular

359 properties were calculated using AlvaDesc (v2.0.10). From the 5666 calculated molecular properties,

3603855 were discarded because they were either not calculated for all molecules or exhibited zero 

estimated by PCA dimensionality reduction on all molecules in $\mathrm{R}$.

\section{Receptor alignment and space estimation}

Mouse ORs were aligned to one another using the MAFFT E-INS-I method with manual refinements ${ }^{43}$.

The resulting alignment file was subjected to ModelTest-NG to identify ideal amino acid substitution scaling were generated from an alignment in which positions with amino acids in at least $60 \%$ of the receptors were considered. Receptor pairwise similarity was calculated by summing amino acid

371 differences at each position by Grantham's amino acid distances ${ }^{17}$. Multidimensional scaling was done 372 in R.

\section{Generating response spectra}

375 To generate odor response spectra, we first began with the $\log _{2} \mathrm{FC}$ values of each odor-responsive OR.

376 Each OR, $r$, was then centered and scaled (z-scored) by mean subtraction and standard deviation

377 division across the odorants, $o$, in the test panel. The resulting matrix is denoted as $\widetilde{\Delta}_{r o}$. To generate

378 property strength vectors, each molecular property, $p$, was z-scored across the odorants in the test

379 panel. The resulting matrix is denoted as $\tilde{P}_{o p}$. To calculate property responses and thereby property response spectra (Pearson correlation coefficients), we used the following formula:

$$
\Phi_{r p}=\sum_{o} \widetilde{\Delta}_{r o} \tilde{P}_{o p}
$$

where $\Phi_{r p}$ refers to Pearson correlation coefficients between individual receptors, $r$, and molecular 
385 To evaluate the significance of the correlation between a property and the response pattern of an OR, 386 we used an FDR cutoff of 0.05. P-values were obtained by first calculating the t-statistic using $t=$

$387 \frac{r \sqrt{n-2}}{\sqrt{1-r^{2}}}$, where $r$ is the correlation coefficient and $n$ is the number of data points. The two-tailed $P$ -

388 value was then calculated as twice the probability a $t$-distributed variable exceeds $t$ using the python 389 scipy.stats.t.sf function. $P$-values were adjusted by FDR correction in R.

391 Odor distance calculation in property space and response space

392 We calculated the distances between odorants in property and response space by calculating Euclidean

393 distances, Pearson correlation coefficients, and cosine similarities between all possible unique pairs of 394 odorants. Molecular properties and receptor response data were normalized to their respective zero 395 mean and unit standard deviation. Correlation distances were reported as $1-r$, and cosine distances 396 were reported as $1-\cos (\theta)$.

\section{Regression models with odor pair cross-validation}

399 Linear models (LASSO and ridge regression) were implemented with the glmnet package ( $v 4.1)$ in $\mathrm{R}^{46}$.

400 XGBoost was implemented with the xgboost module (v1.4.2) in python. Distances (Euclidean, cosine,

401 and correlation) between each unique pair of odorants were calculated in normalized receptor

402 response space. Then, Euclidean distances between each unique pair of odorants were calculated for

403 each feature in normalized feature space. Regularization was then applied as either the L1 (LASSO) or

404 L2 (ridge regression) norm. The $\lambda$ loss function, which controls the number and relative contribution of

405 selected features, was sequentially varied from zero to three by length 1000 . Pearson correlation

406 values were reported for the varied $\lambda$ hyperparameters of the models by comparing model predicted

407 response distances to true response distances. Shuffled controls consisted of using 52 fictitious 
408 odorants whose individual feature vectors were generated by resampling with replacement across the

40952 test odorants.

410

411 Default XGBoost model hyperparameters were used as follows: base_score=0.5, booster='gbtree',

412 colsample_bylevel=1, colsample_bynode=1, colsample_bytree=1, gamma=0, importance_type='gain',

413 interaction_constraints=", learning_rate=0.300000012, max_delta_step=0, max_depth=6,

414 min_child_weight=1, missing=nan, monotone_constraints='()', n_estimators=100,

415 num_parallel_tree=1, random_state=42, reg_alpha=0, reg_lambda=1, scale_pos_weight=1,

416 subsample=1, tree_method='exact', validate_parameters=1, and verbosity=None.

418 Odor pair cross-validation was performed by iteratively holding out each unique pair of odorants from

419 the normalized 52 odor dataset (test data). Features with zero variance from the remaining 50 odor set 420 were dropped (train data). Distances were calculated between the test data for each remaining feature

421 (xtest) and response pattern (ytest). Train data were normalized by z-scoring independent of test data.

422 Distances were then calculated between pairwise combinations of the 50 train odorants for each

423 feature (xtrain) and response pattern (ytrain). Feature distances between the held-out odor pair (xtest)

424 were then used to predict response pattern distances (ypred). Pearson correlation and mean squared

425 error values were reported from comparing model predicted response distances (ypred) to true

426 distances (ytest).

\section{Optimized molecular property selection}

429 To select a subset of molecular properties that were well represented in the data, we utilized Support

430 Vector Machine (SVM) classifiers and regressors with linear kernels in the python sklearn.svm module

431 (v0.24.2). Beginning with the 1811 molecular properties, we first considered those that were non- 
432 continuous (at least one zero entry, ex. molecular weight is a continuous molecular property). Non-

433 zero values were set to one and zero values were kept. Classifiers were trained and cross-validated

434 across the 52 odorant molecules using the normalized 375 deorphanized receptor responses as

435 predictors in a leave-one-out scheme. Data normalization was first performed including the test data.

436 After removing test data, training data were normalized independently to prevent contamination.

437 Classifier area under receiver operating characteristic (AUROC) thresholds of 0.75 were applied.

438 Molecular properties passing this threshold were next subjected to regression with non-zero entries

439 restored. A Pearson correlation cutoff of 0.5 was applied to finally select the 65 "optimized" molecular

440 properties.

441

442 Protein sequence analysis of ORs by logistic regression and SVM classifiers

443 Regularized logistic regression was used to build models linking OR-protein sequence properties to OR-

444 odor responses with the gImnet package (v4.1) in $\mathrm{R}^{46}$. ORs were classified as responders if they

445 exhibited $\log _{2}$ FC $>0$ and FDR $<0.05$ following pS6-IP-Seq and differential expression analysis. For

446 odorants tested at multiple concentrations, the lowest concentration that activated at least 8 ORs was

447 used to promote class stability. Predictors were generated from converting the FASTA alignment file

448 into categorical variables reflecting the presence/absence of specific amino acids at each position.

450 To evaluate model performance, fitted odorants were randomly split into $90 \%$ training and $10 \%$ testing

451 receptor sets. Each test set contained at least one responding receptor. Predictors with zero variance

452 in the training set were dropped. The grid-search optimized $\alpha$ hyperparameter (setting the ratio of the

453 L1 and L2 norms) was set by ten-fold cross-validation with ten-fold cross-validation to set the $\lambda$ (loss

454 function) value. $\lambda$ values one standard error of mean greater than optimal were selected to encourage

455 statistically identical but sparser solutions. Model weighted predictors were then used to determine 
456 the response likelihood of the test receptors. This procedure was repeated 100 times. Non-zero

457 weights were averaged across repetitions and odorants to report positions with residues contributing

458 predictive power towards odor selectivity. WebLogo visualizations were prepared at

459 http://weblogo.threeplusone.com/47

461 SVM classifier response probabilities were calculated using the same inputs as logistic regression using

462100 repetitions of $90 \%$ training (with ten-fold cross-validation for hyperparameter tuning) and $10 \%$

463 testing. Each repetition's response likelihoods and true outcomes were aggregated to generate a single

464 ROC curve for a single odor, which were then combined to generate an aggregate ROC curve.

466 Protein sequence analysis of ORs by comparison to convergently evolved ORs

467 As an alternative strategy, we also performed a statistical evaluation of amino acid properties of ORs

468 sharing responsiveness to an odor against convergently evolved receptors. First, responsive ORs

469 ( $\log _{2} \mathrm{FC}>0$ and FDR $<0.05$ from differential expression) were subset, and pairwise Grantham distances

470 were calculated at each position to generate Grantham distance distributions within the responsive OR

471 alignment. Pairwise comparisons between gaps were considered to have zero distance while pairwise

472 comparisons between gaps and amino acids were considered to have the average Grantham distance

473 across all pairwise comparisons between all ORs at that position. Null distributions were generated

474 similarly from convergently evolved odor-unresponsive ORs. To identify convergently evolved odor-

475 unresponsive sets of ORs, odor-specific receptors with $\log _{2} \mathrm{FC}<0$ or FDR $>0.25$ were first subset. Then,

476 for each unique pairwise comparison between the odor-responsive ORs, full protein sequence

477 Grantham distances were calculated. For each receptor in each pairwise comparison, the closest

478 receptor was selected from the odor-unresponsive subset with the most similar absolute full protein

479 sequence Grantham distance to the pairwise comparison. This meant, for each odor with some 
number of responsive receptors, there was twice as many receptors identified as convergently evolved

481 and odor-unresponsive. Distributions were compared using the Kolmogorov-Smirnov statistical test.

482 FDR correction was applied across all calculated $P$-values with a cutoff of 0.05 . The number of times

483 responding receptors displayed statistically significant deviations in the distribution of Grantham

484 distances from the null set, at each position, was counted and summed across all odorants.

\section{Residue conservation calculation}

487 Using the 313 length alignment file, in which each position was occupied by an amino acid in at least $48860 \%$ of the responsive ORs (387 ORs that were responsive to the lowest concentration of tested 489 odorants yielding response to at least 8 ORs each), we first identified the most common amino acid at 490 each position. We term this the reduced consensus OR sequence. The percent presence of the most 491 commonly occurring amino acid at each position was then reported as conservation percentage for 492 said position.

\section{Homology models}

495 To build an OR homology model, we adapted previously published methods ${ }^{48,49}$. The reduced 496 consensus OR sequence was manually re-aligned to pre-aligned sequences of the bovine rhodopsin 497 (PDB ID 1U19), the human chemokine receptors CXCR4 (3ODU) and CXCR1 (2LNL), and human 498 adenosine A2A receptor (2YDV) using Jalview. Experimental GPCR structures of these receptors were 499 then used as templates to build the homology model of the reduced consensus sequence with 500 Modeller. Visualization and analysis of the homology model was done using VMD and Chimera. 


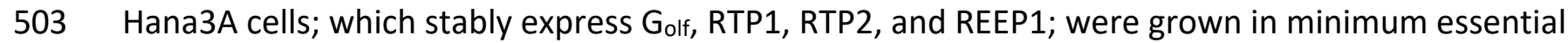

504 medium eagle (MEM; Corning 10-010-CV) containing 10\% Fetal Bovine Serum (FBS; vol/vol; Gibco

505 16000-044), penicillin-streptomycin (Sigma-Aldrich P4333), and amphotericin B (Gibco 15290018). Cells

506 were cultured and incubated at $37^{\circ} \mathrm{C}, 5 \% \mathrm{CO}_{2}$, and saturated humidity for use with the Dual-Glo

507 Luciferase Assay (Promega E2980) ${ }^{29,50}$. Cells were plated at 20-25\% confluence on poly-D-lysine-coated

508 96-well plates (Corning 3843) overnight. After overnight incubation, cells were transfected with $6 \mathrm{~mL}$ of

509 MEM containing 10\% FBS, $0.5 \mu \mathrm{g}$ SV40-RL (Promega E2980), $1 \mu \mathrm{g}$ CRE-Luc (Promega E2980), $0.5 \mu \mathrm{g}$

510 mouse RTP1s, $0.25 \mu \mathrm{g} \mathrm{M3} \mathrm{muscarinic} \mathrm{receptor}{ }^{51}, 0.5 \mu \mathrm{g}$ of Rho-tagged receptor plasmid DNA, and 20

511 ug Lipofectamine 2000 (Invitrogen 11668019) per plate. Transfection medium was divided equally

512 among the wells so that each OR-odorant combination could be conducted in triplicates. The following

513 day, cells were incubated with $25 \mu \mathrm{L}$ of odorant solution diluted in CD-293 (Gibco 11913-019)

514 containing $30 \mu \mathrm{M} \mathrm{CuCl}_{2}$ (Sigma-Aldrich C-6641) and $2 \mathrm{mM}$ glutamine (Gibco 25030-081) for 3.5 hours.

515 cAMP-driven firefly Luciferase luminescence (Luc) was used to assess OR activation, and SV40-driven

516 Renilla Luciferase luminescence (Ren) was used to control for variation in cell viability within wells. Cell

517 luminescence was read by a POLARstar OPTIMA (BMG Labtech) luminometer, and normalized response

518 values were calculated using the formula (Luc-400)/(Ren-400). ORs were considered responsive in vitro

519 if ANOVA $p$-value was $<0.05$ and ANOVA with post-hoc Dunnet's test correction $p$-adjusted was $<0.05$

520 for at least 2 of the tested odor concentrations using the R package DescTools (v0.99.42). Log-logistic

521 4-parameter dose response curves were fit to the data using the R package drc (v3.0-1). In vitro

522 responses were compared to in vivo responses by subtracting mean ligand-independent activity

523 (luciferase values of ORs with no odor stimulation) from each of the ligand stimulated data points and

524 summing. Scaled summed (+)-enantiomer responses were divided by scaled summed (-)-enantiomer

525 responses and $\log _{2}$ transformed for comparison to $\log _{2} \mathrm{FC}(+) /(-)$ in vivo enrichments. 
bioRxiv preprint doi: https://doi.org/10.1101/2021.12.27.474279; this version posted December 28, 2021. The copyright holder for this preprint (which was not certified by peer review) is the author/funder. All rights reserved. No reuse allowed without permission.

\section{Data and code availability:}

528 Data and code are available upon reasonable request. 


\section{$\underline{\text { References: }}$}

5301 Buck, L. \& Axel, R. A novel multigene family may encode odorant receptors: a molecular basis 531 for odor recognition. Cell 65, 175-187, doi:10.1016/0092-8674(91)90418-x (1991).

5322 Hallem, E. A. \& Carlson, J. R. Coding of odors by a receptor repertoire. Cell 125, 143-160, 533 doi:10.1016/j.cell.2006.01.050 (2006).

5343 Wang, G., Carey, A. F., Carlson, J. R. \& Zwiebel, L. J. Molecular basis of odor coding in the malaria vector mosquito Anopheles gambiae. Proc Natl Acad Sci U S A 107, 4418-4423, doi:10.1073/pnas.0913392107 (2010). Carey, A. F., Wang, G., Su, C. Y., Zwiebel, L. J. \& Carlson, J. R. Odorant reception in the malaria mosquito Anopheles gambiae. Nature 464, 66-71, doi:10.1038/nature08834 (2010). Saito, H., Chi, Q., Zhuang, H., Matsunami, H. \& Mainland, J. D. Odor coding by a Mammalian receptor repertoire. Sci Signal 2, ra9, doi:10.1126/scisignal.2000016 (2009). Keller, A. et al. Predicting human olfactory perception from chemical features of odor molecules. Science 355, 820-826, doi:10.1126/science.aal2014 (2017). $\mathrm{Xu}$, L. et al. Widespread receptor-driven modulation in peripheral olfactory coding. Science 368, doi:10.1126/science.aaz5390 (2020). Zhao, H. et al. Functional expression of a mammalian odorant receptor. Science 279, 237-242, doi:10.1126/science.279.5348.237 (1998). Araneda, R. C., Kini, A. D. \& Firestein, S. The molecular receptive range of an odorant receptor. Nat Neurosci 3, 1248-1255, doi:10.1038/81774 (2000). Malnic, B., Hirono, J., Sato, T. \& Buck, L. B. Combinatorial receptor codes for odors. Cell 96, 713723, doi:10.1016/s0092-8674(00)80581-4 (1999). Jiang, Y. et al. Molecular profiling of activated olfactory neurons identifies odorant receptors for odors in vivo. Nat Neurosci 18, 1446-1454, doi:10.1038/nn.4104 (2015). von der Weid, B. et al. Large-scale transcriptional profiling of chemosensory neurons identifies receptor-ligand pairs in vivo. Nat Neurosci 18, 1455-1463, doi:10.1038/nn.4100 (2015). Knight, Z. A. et al. Molecular profiling of activated neurons by phosphorylated ribosome capture. Cell 151, 1126-1137, doi:10.1016/j.cell.2012.10.039 (2012). $\mathrm{Hu}, \mathrm{X}$. S. et al. Concentration-Dependent Recruitment of Mammalian Odorant Receptors. eNeuro 7, doi:10.1523/eneuro.0103-19.2019 (2020). plastic. Elife 9, doi:10.7554/elife.54501 (2020).

Ravia, A. et al. A measure of smell enables the creation of olfactory metamers. Nature 588,118 123, doi:10.1038/s41586-020-2891-7 (2020).

1 Grantham, R. Amino acid difference formula to help explain protein evolution. Science 185, 862-864, doi:10.1126/science.185.4154.862 (1974).

Chae, H. et al. Mosaic representations of odors in the input and output layers of the mouse olfactory bulb. Nat Neurosci 22, 1306-1317, doi:10.1038/s41593-019-0442-z (2019).

9 Pashkovski, S. L. et al. Structure and flexibility in cortical representations of odour space. Nature 583, 253-258, doi:10.1038/s41586-020-2451-1 (2020).

20 Zhou, Q. et al. Common activation mechanism of class A GPCRs. Elife 8, doi:10.7554/eLife.50279 (2019).

21 Liapakis, G. et al. The forgotten serine. A critical role for Ser-2035.42 in ligand binding to and activation of the beta 2-adrenergic receptor. J Biol Chem 275, 37779-37788, doi:10.1074/jbc.M002092200 (2000). 
57422 Strader, C. D., Candelore, M. R., Hill, W. S., Sigal, I. S. \& Dixon, R. A. Identification of two serine

575

576

577

578

579

580

581

582

583

584

585

586

587

588

589

590

591

592

593

594

595

596

597

598

599

600

601

602

603

604

605

606

607

608

609

610

611

612

613

614

615

616

617

619

620

residues involved in agonist activation of the beta-adrenergic receptor. J Biol Chem 264, 1357213578 (1989).

23 Katritch, V. \& Abagyan, R. GPCR agonist binding revealed by modeling and crystallography. Trends Pharmacol Sci 32, 637-643, doi:10.1016/j.tips.2011.08.001 (2011).

24 Munk, C., Harpsoe, K., Hauser, A. S., Isberg, V. \& Gloriam, D. E. Integrating structural and mutagenesis data to elucidate GPCR ligand binding. Curr Opin Pharmacol 30, 51-58, doi:10.1016/j.coph.2016.07.003 (2016).

25 Surgand, J. S., Rodrigo, J., Kellenberger, E. \& Rognan, D. A chemogenomic analysis of the transmembrane binding cavity of human G-protein-coupled receptors. Proteins 62, 509-538, doi:10.1002/prot.20768 (2006).

26 Shi, L. et al. Beta2 adrenergic receptor activation. Modulation of the proline kink in transmembrane 6 by a rotamer toggle switch. J Biol Chem 277, 40989-40996, doi:10.1074/jbc.M206801200 (2002).

27 Eddy, M. T. et al. Allosteric Coupling of Drug Binding and Intracellular Signaling in the A2A Adenosine Receptor. Cell 172, 68-80 e12, doi:10.1038/nbt.4096 (2018).

28 de March, C. A. et al. Conserved Residues Control Activation of Mammalian G Protein-Coupled Odorant Receptors. J Am Chem Soc 137, 8611-8616, doi:10.1021/jacs.5b04659 (2015).

29 Saito, H., Kubota, M., Roberts, R. W., Chi, Q. \& Matsunami, H. RTP family members induce functional expression of mammalian odorant receptors. Cell 119, 679-691, doi:10.1016/j.cell.2004.11.021 (2004).

30 Larsson, M. C. et al. Or83b encodes a broadly expressed odorant receptor essential for Drosophila olfaction. Neuron 43, 703-714, doi:10.1016/j.neuron.2004.08.019 (2004).

31 Sanchez-Lengeling, B. et al. Machine Learning for Scent: Learning Generalizable Perceptual Representations of Small Molecules. arXiv:1910.10685 (2019). <https://ui.adsabs.harvard.edu/abs/2019arXiv191010685S>.

32 Tran, N., Kepple, D., Shuvaev, S. \& Koulakov, A. in Proceedings of the 36th International Conference on Machine Learning Vol. 97 (eds Chaudhuri Kamalika \& Salakhutdinov Ruslan) 6305--6314 (PMLR, Proceedings of Machine Learning Research, 2019).

33 Katada, S., Hirokawa, T., Oka, Y., Suwa, M. \& Touhara, K. Structural basis for a broad but selective ligand spectrum of a mouse olfactory receptor: mapping the odorant-binding site. $J$ Neurosci 25, 1806-1815, doi:10.1523/jneurosci.4723-04.2005 (2005).

$34 \mathrm{Yu}, \mathrm{Y}$. et al. Responsiveness of $\mathrm{G}$ protein-coupled odorant receptors is partially attributed to the activation mechanism. Proc Natl Acad Sci U S A 112, 14966-14971, doi:10.1073/pnas.1517510112 (2015).

35 Sekharan, S. et al. QM/MM model of the mouse olfactory receptor MOR244-3 validated by sitedirected mutagenesis experiments. Biophys J 107, L5-I8, doi:10.1016/j.bpj.2014.07.031 (2014).

36 Baud, O. et al. Exchanging ligand-binding specificity between a pair of mouse olfactory receptor paralogs reveals odorant recognition principles. Sci Rep 5, 14948, doi:10.1038/srep14948 (2015).

37 Gelis, L., Wolf, S., Hatt, H., Neuhaus, E. M. \& Gerwert, K. Prediction of a ligand-binding niche within a human olfactory receptor by combining site-directed mutagenesis with dynamic homology modeling. Angew Chem Int Ed Eng/ 51, 1274-1278, doi:10.1002/anie.201103980 (2012). olfactory receptors. Nature, doi:10.1038/s41586-021-03794-8 (2021). 
62240 Li, B. \& Dewey, C. N. RSEM: accurate transcript quantification from RNA-Seq data with or

623 without a reference genome. BMC Bioinformatics 12, 323, doi:10.1186/1471-2105-12-323

624 (2011).

62541 Robinson, M. D., McCarthy, D. J. \& Smyth, G. K. edgeR: a Bioconductor package for differential expression analysis of digital gene expression data. Bioinformatics 26, 139-140,

62842 Smedley, D. et al. BioMart--biological queries made easy. BMC Genomics 10, 22,

629 doi:10.1186/1471-2164-10-22 (2009).

63043 Katoh, K., Kuma, K., Toh, H. \& Miyata, T. MAFFT version 5: improvement in accuracy of multiple sequence alignment. Nucleic Acids Res 33, 511-518, doi:10.1093/nar/gki198 (2005). Darriba, D. et al. ModelTest-NG: A New and Scalable Tool for the Selection of DNA and Protein Evolutionary Models. Mol Biol Evol 37, 291-294, doi:10.1093/molbev/msz189 (2020).

45 Kozlov, A. M., Darriba, D., Flouri, T., Morel, B. \& Stamatakis, A. RAxML-NG: a fast, scalable and user-friendly tool for maximum likelihood phylogenetic inference. Bioinformatics 35, 44534455, doi:10.1093/bioinformatics/btz305 (2019). Friedman, J., Hastie, T. \& Tibshirani, R. Regularization Paths for Generalized Linear Models via Coordinate Descent. J Stat Softw 33, 1-22 (2010).

63947 Crooks, G. E., Hon, G., Chandonia, J. M. \& Brenner, S. E. WebLogo: a sequence logo generator. Genome Res 14, 1188-1190, doi:10.1101/gr.849004 (2004).

49 de March, C. A., Kim, S. K., Antonczak, S., Goddard, W. A., 3rd \& Golebiowski, J. G protein-

651

51 Li, Y. R. \& Matsunami, H. Activation state of the M3 muscarinic acetylcholine receptor modulates mammalian odorant receptor signaling. Sci Signal 4, ra1, doi:10.1126/scisignal.2001230 (2011). 